NASA Technical Memorandum 4775

\title{
Development of a Closed-Loop Strap Down Attitude System for an Ultrahigh Altitude Flight Experiment
}

Stephen A. Whitmore, Mike Fife, and Logan Brashear

January 1997

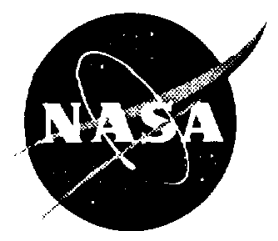


NASA Technical Memorandum 4775

\section{Development of a Closed-Loop Strap Down Attitude System for an Ultrahigh Altitude Flight Experiment}

Stephen A. Whitmore

Dryden Flight Research Center

Edwards, California

Mike Fife

Massachusetts Institute of Technology

Cambridge, Massachusetts

Logan Brashear

University of California

Los Angeles, California

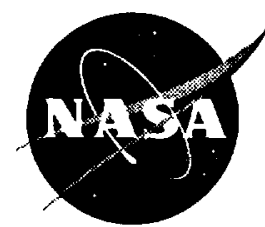

National Aeronautics and

Space Administration

Office of Management

Scientific and Technical

Information Program

1997 



\title{
DEVELOPMENT OF A CLOSED-LOOP STRAP DOWN ATTITUDE SYSTEM FOR AN ULTRAHIGH ALTITUDE FLIGHT EXPERIMENT
}

\author{
Stephen A. Whitmore \\ NASA Dryden Flight Research Center \\ Edwards, California \\ Mike Fife \\ Massachusetts Institute of Technology \\ Cambridge, Massachusetts \\ Logan Brashear \\ University of California \\ Los Angeles, California
}

\begin{abstract}
$\underline{\text { Abstract }}$
A low-cost attitude system has been developed for an ultrahigh altitude flight experiment. The experiment uses a remotely piloted sailplane, with the wings modified for flight at altitudes greater than 100,000 ft. Mission requirements deem it necessary to measure the aircraft pitch and bank angles with accuracy better than $1.0^{\circ}$ and heading with accuracy better than $5.0^{\circ}$. Vehicle cost restrictions and gross weight limits make installing a commercial inertial navigation system unfeasible. Instead, a low-cost attitude system was developed using strap down components. Monte Carlo analyses verified that two vector measurements, magnetic field and velocity, are required to completely stabilize the error equations. In the estimating algorithm, body-axis observations of the airspeed vector and the magnetic field are compared against the inertial velocity vector and a magnetic-field reference model. Residuals are fed back to stabilize integration of rate gyros. The effectiveness of the estimating algorithm was demonstrated using data from the NASA Dryden Flight Research Center Systems Research Aircraft (SRA) flight tests. The algorithm was applied with good results to a maximum $10^{\circ}$ pitch and

\footnotetext{
"Aerospace Engineer, Senior Member AIAA

${ }^{\dagger}$ Graduate Student, Student Member AIAA

${ }^{\ddagger}$ Graduate Student

Copyright (1) 1997 by the American Institute of Aeronautics and
} Astronautics, Inc. No copyright is asserted in the United States under Title 17, U.S. Code. The U.S. Government has a royalty-free license to exercise all rights under the copyright claimed herein for Governmental purposes. All other rights are reserved by the copyright owner.
\end{abstract}

bank angles. Effects of wind shears were evaluated and, for most cases, can be safely ignored.

\section{Nomenclature}

$b$

$c$

$d$

d

E expectation operator

$F \quad$ state equation matrix function, deg

$G_{U_{k+1}} \quad$ airdata error covariance matrix, $(\mathrm{ft} / \mathrm{sec})^{2}$

$G_{V_{k+1}} \quad$ inertial velocity error covariance matrix, $(\mathrm{ft} / \mathrm{sec})^{2}$

$G_{Z_{k+1}} \quad$ magnetometer error covariance matrix, (micro-Tesla) $)^{2}$

$H \quad$ altitude, $\mathrm{ft}$

I identity matrix

$I_{p} \quad$ time integral of roll rate, deg

$I_{q} \quad$ time integral of pitch rate, deg

$I_{\mathrm{r}} \quad$ time integral of yaw rate, deg

$K \quad$ airdata/velocity correction gain matrix

$k \quad$ time index

lat latitude, deg north

long longitude, deg west 


\begin{tabular}{|c|c|}
\hline$M[]$. & direction cosine matrix \\
\hline$m_{i, j}$ & $\mathrm{i}, \mathrm{j}^{\text {th }}$ component of direction cosine matrix \\
\hline$P_{k x}$ & $\begin{array}{l}\text { state error covariance matrix, previous } \\
\text { data frame, } \mathrm{deg}^{2}\end{array}$ \\
\hline$P_{k+1 / k}$ & $\begin{array}{l}\text { predicted state error covariance } \\
\text { matrix }, \mathrm{deg}^{2}\end{array}$ \\
\hline$P_{k+1 / k+1}$ & $\begin{array}{l}\text { corrected state error covariance } \\
\text { matrix } \operatorname{deg}^{2}\end{array}$ \\
\hline$p$ & roll rate, $\mathrm{deg} / \mathrm{sec}$ \\
\hline$q$ & pitch rate, $\mathrm{deg} / \mathrm{sec}$ \\
\hline$Q_{k+1}$ & $\begin{array}{l}\text { state equation error covariance } \\
\text { matrix, }(\mathrm{deg} / \mathrm{sec})^{2}\end{array}$ \\
\hline$Q_{x}$ & quaternion vector \\
\hline$\left\|Q_{x}\right\|$ & norm of the quaternion vector \\
\hline$r$ & yaw rate, $\mathrm{deg} / \mathrm{sec}$ \\
\hline$T$ & $\begin{array}{l}\text { magnetic field reference datum, } \\
\text { micro-tesla }\end{array}$ \\
\hline$t$ & time, sec \\
\hline$t_{0}$ & initial time, sec \\
\hline$U$ & airspeed vector, $\mathrm{ft} / \mathrm{sec}$ \\
\hline$V$ & inertial velocity vector, $\mathrm{ft} / \mathrm{sec}$ \\
\hline$W$ & wind velocity vector, $\mathrm{ft} / \mathrm{sec}$ \\
\hline$Z$ & magnetometer measurement, micro-tesla \\
\hline$\alpha$ & angle of attack, deg \\
\hline$\beta$ & angle of sideslip, deg \\
\hline$\delta$ & vector error, deg \\
\hline$\Delta t$ & sample interval, sec \\
\hline$\eta$ & angular velocity matrix, $\mathrm{deg} / \mathrm{sec}$ \\
\hline$\Theta$ & attitude vector, deg \\
\hline$\theta$ & pitch angle, deg. \\
\hline$\kappa$ & magnetometer correction gain matrix \\
\hline$\Phi$ & state transition matrix \\
\hline$\phi$ & bank angle, deg. \\
\hline$\psi$ & yaw angle, deg. \\
\hline$\Omega$ & integrating factor matrix, deg \\
\hline$\omega$ & angular velocity vector, $\mathrm{deg} / \mathrm{sec}$ \\
\hline$\|\omega\|$ & norm of the angular velocity integral, deg \\
\hline$\nabla$ & gradient vector \\
\hline
\end{tabular}

\section{Superscripts and subscripts}

[ ] vertical (+ down) component of Earth-axis vector

[ ] east component of Earth-axis vector

[]$_{k / k} \quad$ state estimate from previous data frame

[]$_{k+1 / k} \quad$ state estimate-based open-loop integration

[]$_{k+1 / k+1} \quad$ state estimate after correction by magnetometer or velocity data

[ ] lat lateral component of body-axis vector

[ ] long longitudinal component of body-axis vector

[]$_{n} \quad$ north component of Earth-axis vector

[]$_{\text {norm }}$ normal component of body-axis vector

[]$^{T} \quad$ matrix transpose

[] state estimate after magnetometer correction

[] time derivative, $1 / \mathrm{sec}$

\section{Acronyms}

ADC airdata computer

DFRC Dryden Flight Research Center

FCS flight control system

GPS Global Positioning System

INS inertial navigation system

MOS model output statistics

RT-FADS real-time flush airdata sensing

SRA Systems Research Aircraft

\section{Introduction}

Interest in ultrahigh altitude aircraft for atmospheric sampling and remote Earth-sensing is growing, and several aircraft are currently in the process of proving the feasibility of extended duration flight to a maximum altitude of $80,000 \mathrm{ft}$. Requirements for flights as high as an altitude of $120,000 \mathrm{ft}$ have been identified. These flight regimes are difficult to design for, because research into low-Reynolds number, high-subsonic aerodynamics has been very limited. Although some basic airfoils for this flight regime have been analyzed and tested in wind tunnels, ${ }^{1.2}$ fundamental data on entire vehicle aerodynamics, flight mechanics, and flight performance are lacking. 
A preliminary design study was undertaken at the NASA Dryden Flight Research Center (DFRC) recently ${ }^{3}$ with the objective of finding a satisfactory method for achieving trimmed flight at an altitude of $100,000 \mathrm{ft}$. The study for a high-altitude flight experiment examined several possible techniques for achieving this objective. The study examined the feasibility of using a highaltitude balloon to tow a remotely piloted sailplane to an altitude of $100,000 \mathrm{ft}$, where it would be released and flown back to a lakeside landing on Rogers dry lakebed at Edwards Air Force Base, California.

The preliminary study concluded that the most feasible approach is to use a commercially available sailplane, with the wings modified for the low-Reynolds number, high-subsonic Mach number flight. For the ultrahigh altitude flight experiment, no propulsive power plant exists and this provides a low-noise environment for studying low-Reynolds number transition phenomena. A portion of the unswept, untapered wing will serve as a test section for examining flow transition physics under these conditions. Figure 1 shows the ultrahigh altitude vehicle.

The current mission plan requires a nosedown balloon release, with booster rockets used to generate pseudolift to turn the vehicle from its nosedown configuration to level flight. After sufficient Mach number, (approximately Mach 0.65) for free flight at high altitudes has been achieved, the rocket pack is jettisoned. At launch and during the transition maneuver, accelerations along the vertical axis are substantial. Figure 2 shows a schematic of the mission concept.

The unpowered aircraft will collect airfoil aerodynamics and vehicle performance data from launch to a lakebed landing at Edwards Air Force Base. The principal research objectives of the ultrahigh altitude flight experiment program are:

1. To validate high-altitude airfoil design methodologies by measuring airfoil and vehicle characteristics at low Reynolds numbers and high subsonic Mach numbers in a low-turbulence flight environment.

2. To establish a test bed aircraft for ultrahigh altitude flight research.

Onboard measurements include boundary-layer velocity profiles from total pressure rakes at several streamwise locations, chordwise pressure distributions, the boundary-layer laminar-to-turbulent transition state, airfoil section drag (fixed wake rake), and flight mechanics data such as local angle of attack, free-stream airdata, linear accelerations, angular rates, and the aircraft attitudes. The experiment will use an onboard data acquisition system, and data will be telemetered to

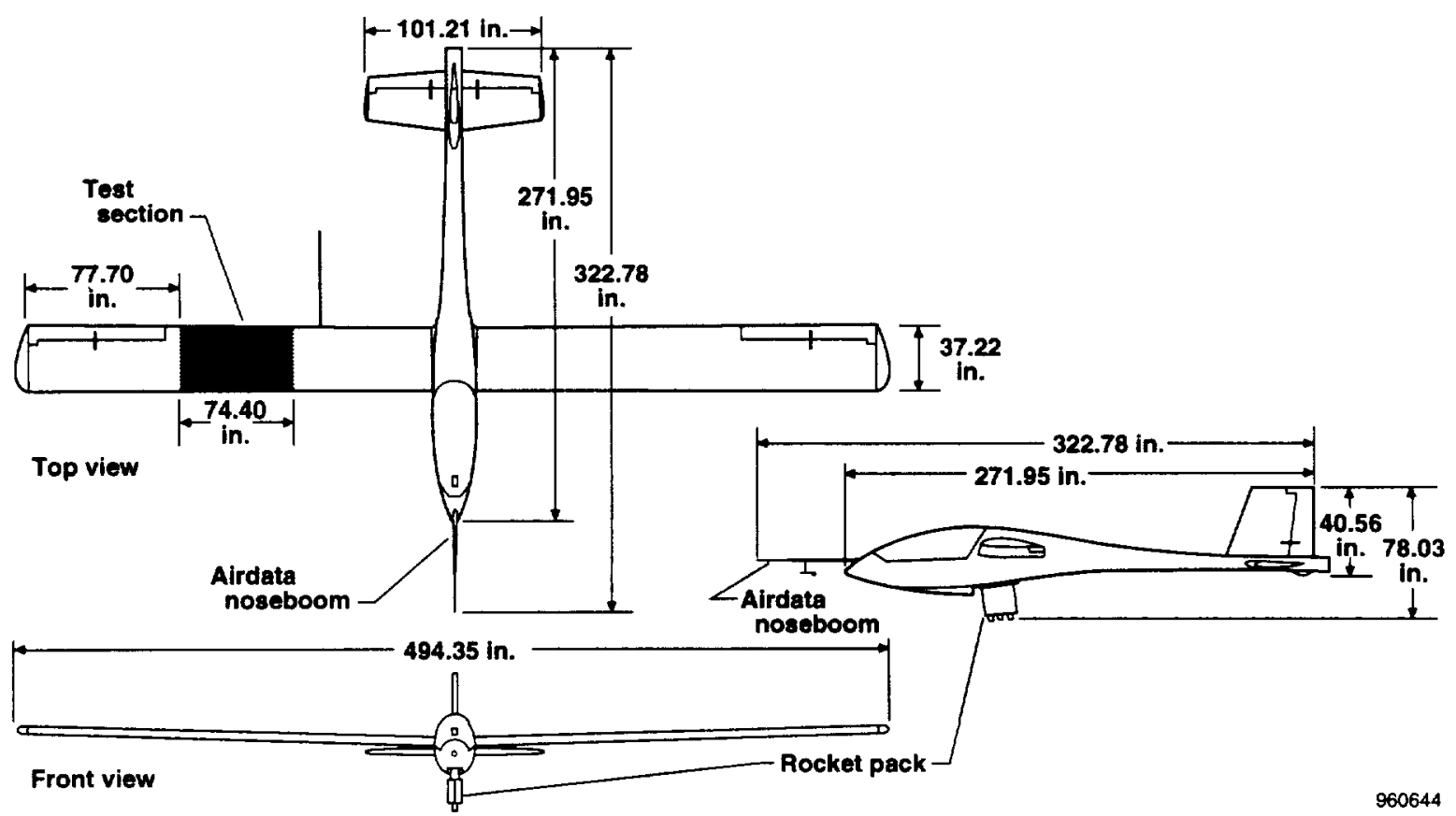

Figure 1. The ultrahigh altitude vehicle. 


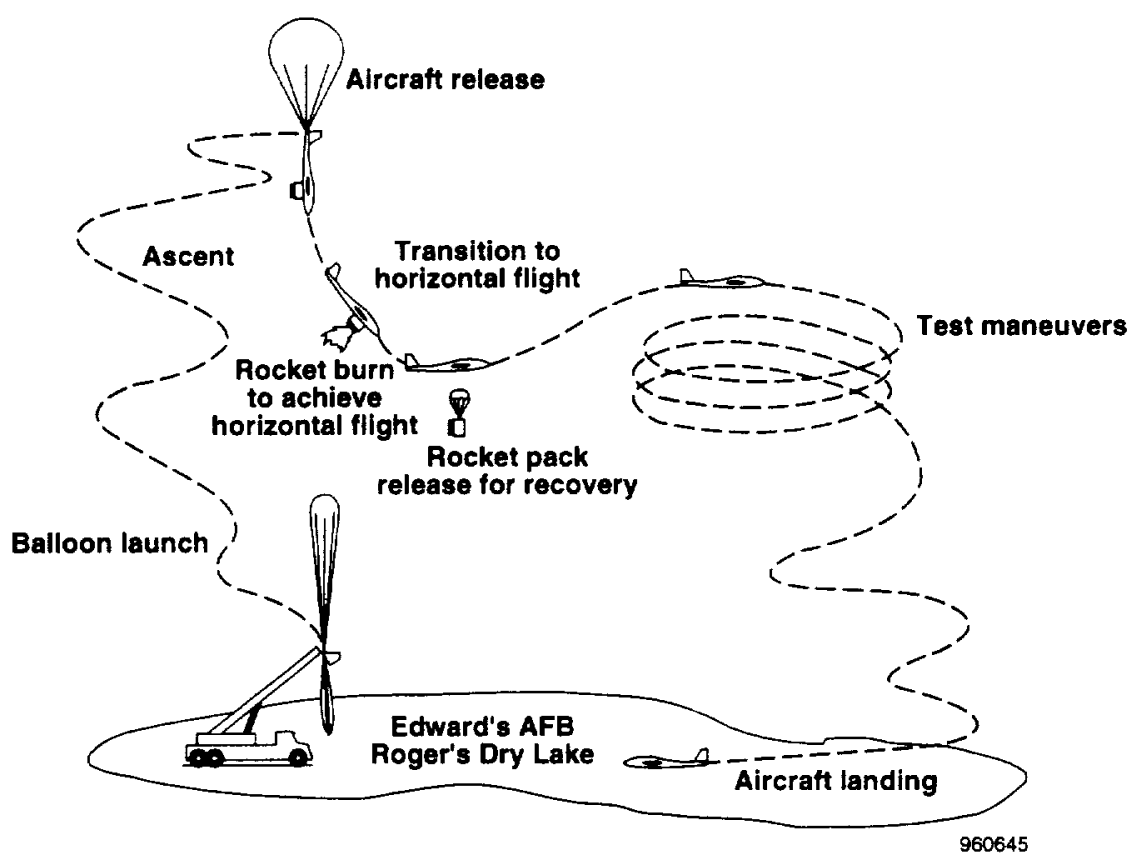

Figure 2. Schematic of mission concept.

a ground-based recording station. Flight test maneuvers will consist of stabilized turns to achieve higher than 1-g trim angles of attack and constant lift coefficient or Mach-number descents.

To accomplish the research objectives of this experiment, measuring the absolute pitch and roll orientation of the aircraft to better than $1.0^{\circ}$ and a resolution of $0.5^{\circ}$ at the high altitudes is necessary. For pilot navigation, measuring the vehicle heading with an absolute accuracy of better than $5.0^{\circ}$ with a resolution of $1.0^{\circ}$ is desirable. These attitude requirements result from the narrow speed range allowable along the flight envelope at these extreme altitudes. Flying too fast causes the airfoil to develop shocks, causing potential separation and loss of lift. At excessive speeds, a flutter boundary may also be approached. Flying too slowly causes the aircraft to approach stall speed. Typically, an improper attitude will translate into an unacceptable speed change within approximately $5-10 \mathrm{sec}$. This speed change either drives the pilot into a "speed-induced" longitudinal oscillation or reduces the pilot's attention to nothing but speed control. For this program the tolerance requirements for the test points are restrictive, and precise attitude information is required to ensure that multiple flight conditions can be met simultaneously.

The stated requirements for attitude and heading could be achieved using state-of-the-art inertial navigation systems, both gimballed and strap down, ${ }^{4}$ but the costs of these systems are considered excessive for the ultrahigh altitude flight experiment program. Gross vehicle weight at high altitudes is of concern, and the extra weight penalty caused by adding a full inertial navigation system (INS) was considered undesirable. Furthermore, the desired accuracy requirements will not be met by open-loop integration of strap-down rate gyros.

To circumvent this problem, a simple lightweight, low-power consumption, strap-down attitude system concept was developed for this program and is detailed in this paper. In this system concept, body-axis observations of the airspeed and magnetic-field vectors are compared with the known measured inertial velocity vector and known magnetic-field vectors (in Earthrelative coordinates) to provide a "virtually inertial" reference that is used to infer an attitude error. This error is then fed back to correct and stabilize the rate-gyro integration. The system has the stability of gimballed attitude systems, but relies on low-cost strap-down components to gather the required information. The system performance is analyzed for the launch trajectory using Monte Carlo ${ }^{5}$ error simulations. Effects of instrumentation bias and random errors are analyzed. Particular attention is paid to what type of feedback is required to ensure full-loop closure and, hence, algorithm stability. 
Data derived from the NASA Dryden System Research Aircraft (SRA) flight tests ${ }^{6}$ are used to demonstrate the effectiveness of the estimating algorithm. The algorithm was applied to data from several flights with good results achieved for up to $10^{\circ}$ pitch and roll attitude. Reasons for estimate degradation at high attitudes are discussed. Effects of wind shears on attitude estimates are evaluated using rawindsonde weather balloon measurements.

\section{Coordinate Definitions}

Figure 3 shows the coordinate definitions used in this report. The Euler angles describe the aircraft body-axis orientation (in longitudinal, lateral and normal coordinates, with respect to the local tangent plane of the Earth and true north) in north, east, and down coordinates. The direction cosine matrix that allows transformation from Earth axis to body axis, is the product of three successive rotations: ${ }^{6}$
Here, $\theta$ is the pitch angle, $\phi$ is the roll rate or bank angle, and $\psi$ is the yaw or heading angle. The angular velocity of the aircraft is typically measured by gyroscopic instruments fixed to the aircraft body axis. The angular velocity vector ( $p$ is the roll rate, $q$ is the pitch rate, and $r$ is the yaw rate) expressed in body axis, is related to Earth axis by a transformation similar to equation $1:^{6}$

$$
\left[\begin{array}{c}
\dot{\phi} \\
\dot{\theta} \\
\dot{\psi}
\end{array}\right]=\frac{1}{\cos \theta}\left[\begin{array}{ccc}
\cos \theta & (\sin \phi \sin \theta) & \cos \phi \sin \theta \\
0 & \cos \phi \cos \theta & -\sin \phi \cos \theta \\
0 & \sin \phi & \cos \phi
\end{array}\right]\left[\begin{array}{l}
p \\
q \\
r
\end{array}\right]
$$

\section{Proposed Attitude System}

Equation 3 can be directly integrated to give the Euler angles, given a known initial condition. But because equation 3 is neutrally stable, bias or systematic errors in

$$
\begin{aligned}
M[\theta, \phi, \psi] & =\left[\begin{array}{ccc}
1 & 0 & 0 \\
0 & \cos \phi & \sin \phi \\
0 & -\sin \phi & \cos \phi
\end{array}\right]\left[\begin{array}{ccc}
\cos \theta & 0 & -\sin \theta \\
0 & 1 & 0 \\
\sin \theta & 0 & \cos \theta
\end{array}\right]\left[\begin{array}{ccc}
\cos \psi & \sin \psi & 0 \\
-\sin \psi & \cos \psi & 0 \\
0 & 0 & 1
\end{array}\right] \\
& =\left[\begin{array}{c}
(\cos \theta \cos \psi) \\
(\sin \phi \sin \theta \cos \psi-\cos \phi \sin \psi) \\
(\cos \phi \sin \theta \cos \psi+\sin \phi \sin \psi) \\
(\sin \theta \sin \psi+\cos \phi \cos \psi)(\sin \phi \cos \theta)
\end{array}\right]=\left\{m_{i, j}\right\}
\end{aligned}
$$

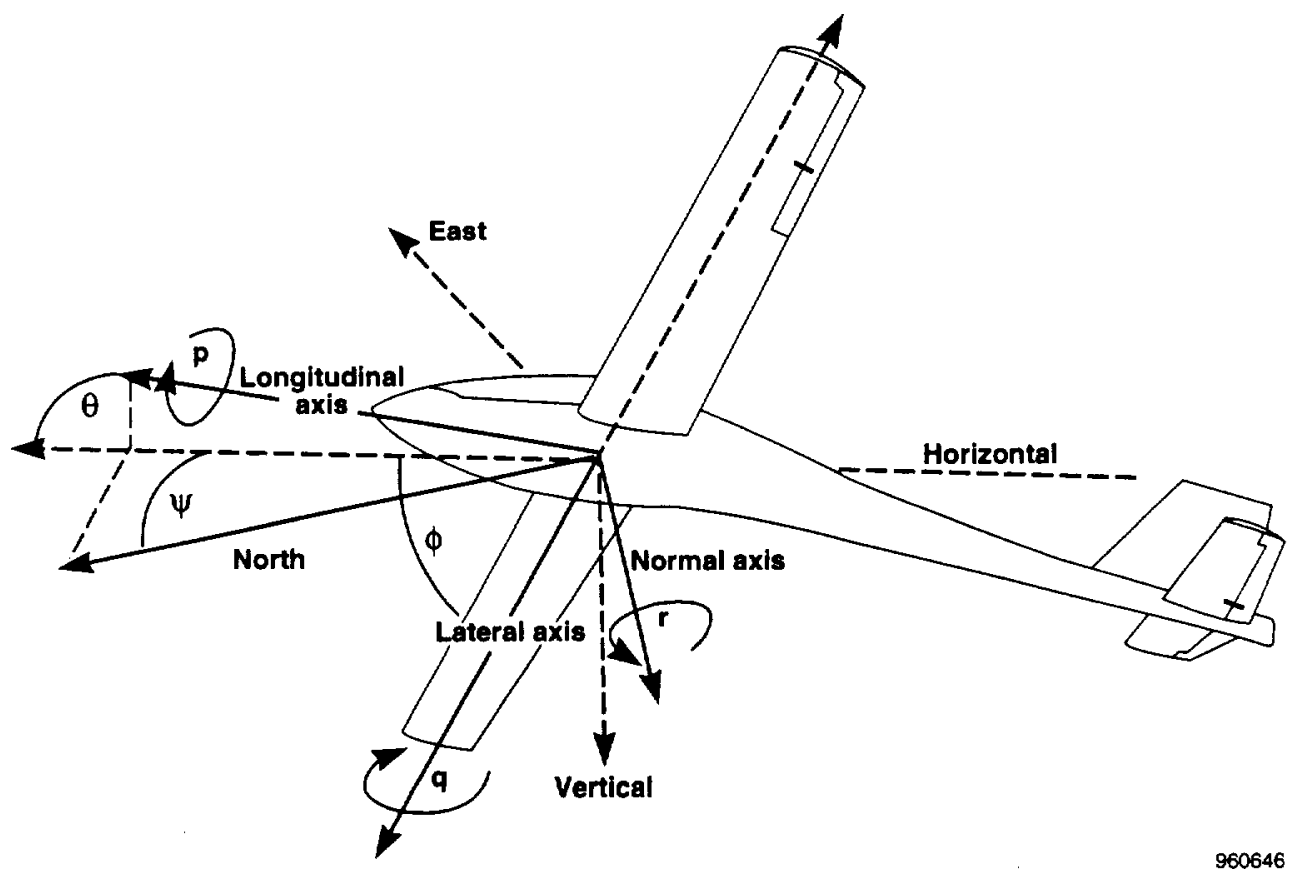

Figure 3. Axes and coordinate definitions. 
the rate-gyro measurements cause the integration to drift from the true attitudes as a function of time. Thus, loop closure is required to get a stable attitude measurement. For conventional inertial systems, a stable reference is provided by a gimballed platform. Payload limitations of the vehicle and large vertical accelerations at launch make this approach unfeasible for this ultrahigh altitude flight experiment, as described earlier.

The system proposed for the ultrahigh altitude vehicle uses strap-down components that are low in cost, do not require a stable member to perform the integration, and are insensitive to large accelerations in the vertical axes. Body-axis angular rates are integrated and stabilized using measurements of airspeed, inertial velocity, and magnetic-field vectors. As will be shown in the results and discussion section, two vector measurements, magnetic field and velocity, are required to achieve complete three-attitude stability. The approach compares body-axis observations of the magnetic field with a magnetic-field datum derived from a mathematical model $^{7}$ and the airspeed vector with the inertially sensed velocity to infer an attitude error. This error signal is fed back to stabilize the gyro integration. Because closed-loop stability is achieved with strap-down components, this system may be referred to as a "virtually inertial" attitude system. Table 1 shows the list of required measurements, the data sources, and approximate sample rates at which the data will be used for typical applications.

Required accuracy for the various sensing components will be developed by the simulation studies to be presented in the results and discussion section. Figure 4 shows the basic system layout along with the structure of the estimation algorithm (to be developed in the next section).

Table 1. List of required measurements.

\begin{tabular}{lllc}
\hline \hline Measurement & Axis system & Source & Update rate \\
\hline Angular rates, $p, q, r$ & body axis & Rate gyro, 3-axis & $20-50 \mathrm{~Hz}$ \\
Magnetic field vector, $Z$ & body axis & 3-axis magnetometer & $1 \mathrm{~Hz}$ \\
Airdata, $U_{\infty}, \alpha, \beta$ & body axis & Airdata probe/boom & $1-10 \mathrm{~Hz}$ \\
Inertial velocity, $V_{n}, V_{e}, V_{d}$ & Earth axis & Global Positioning System (GPS) & $1-10 \mathrm{~Hz}$ \\
Aircraft lat, long, $H$ & Earth axis & Global Positioning System (GPS) & $1 \mathrm{~Hz}$ \\
Magnetic field datum, $T$ & Earth axis & Spherical harmonic model & $1 \mathrm{~Hz}$ \\
\hline \hline
\end{tabular}

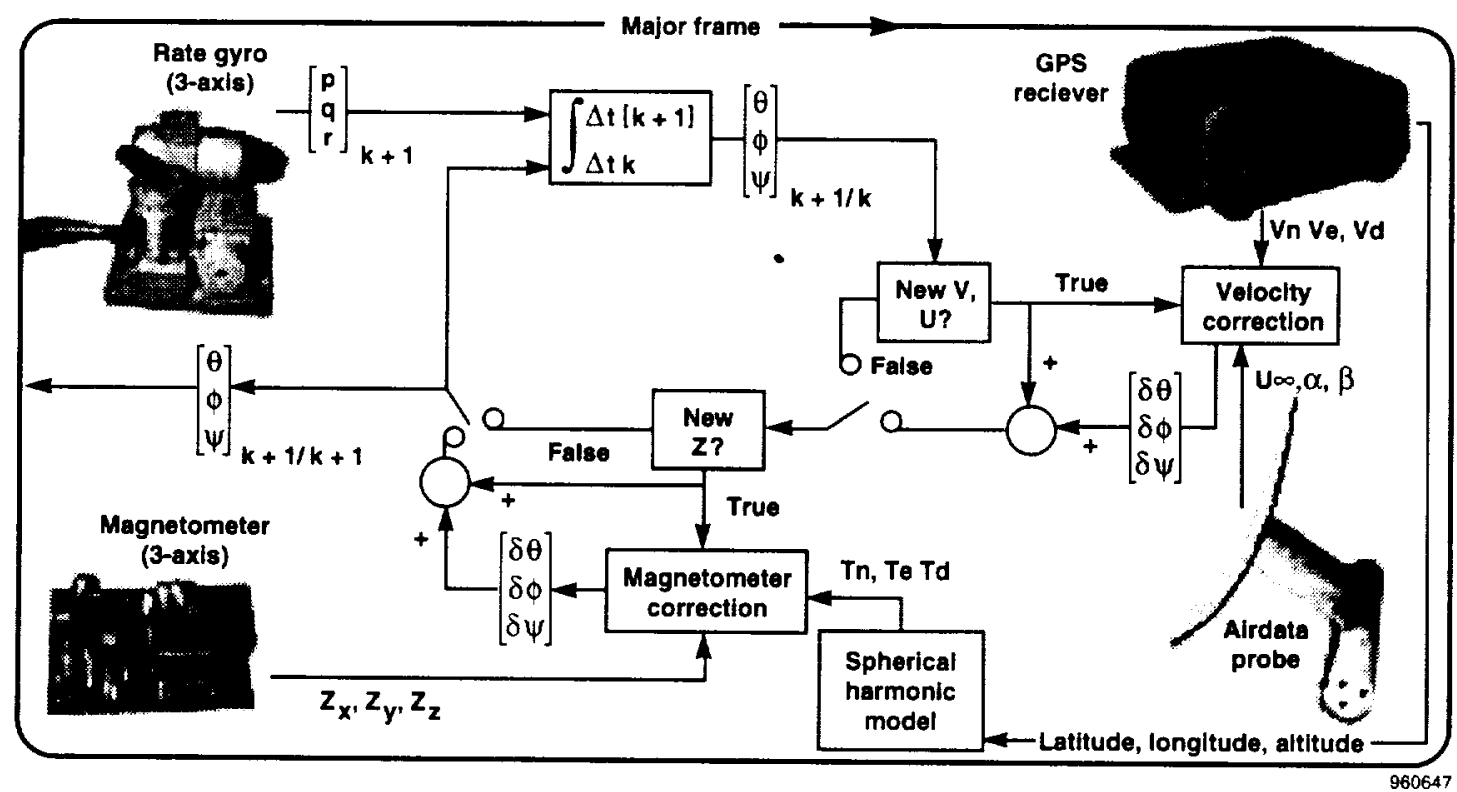

Figure 4. Schematic of the ultrahigh altitude flight experiment attitude system. 


\section{Development of the Estimation Algorithm}

This section develops a closed-loop estimation algorithm for the strap-down (nongimballed) attitude system. Measurement errors from the independent systems, (such as airspeed, magnetometer, inertial velocity, and angular rates) are assumed to be uncorrelated. The algorithm emulates the form of the Kalman filter, modified for asynchronously arriving data (the fundamental Kalman assumes that all data from the various measurement sources arrive simultaneously). For the types of disparate data sources to be used for ultrahigh altitude flight systems (for example, magnetometer, Global Positioning System (GPS), analog), a frame-by-frame algorithmic approach is too restrictive. To circumvent this difficulty, the estimation algorithm is implemented as a two-step predictor/ corrector filter where the prediction-step (quaternion integration of the rate-gyro data) is performed at a fixed rate, typically $25-50 \mathrm{~Hz}$, and the correction step is performed whenever fresh velocity and magnetometer data are available, typically $1-10 \mathrm{~Hz}$.

\section{Quaternion Formulation of the Rate Integrator}

As is typical of all modern INS systems, to eliminate problems with infinite angular rates caused by the nosedown initial attitude at launch, the estimation algorithm formulates the problem in terms of quaternion parameters. ${ }^{4.8}$ In the quaternion transformation the orientation is written as a 4-space vector with the magnitude being constrained to always be unity. Using $m_{i, j}$ for the elements of the direction cosine matrix (eq. 2), then the quaternions can be evaluated by

$$
Q_{x} \equiv\left[\begin{array}{l}
a \\
b \\
c \\
d
\end{array}\right]=\left[\begin{array}{c}
\frac{m_{23}-m_{32}}{4 d} \\
\frac{m_{31}-m_{13}}{4 d} \\
\frac{m_{12}-m_{21}}{4 d} \\
\frac{1}{2} \sqrt{1+m_{11}+m_{22}+m_{33}}
\end{array}\right] \Rightarrow Q_{x} \|^{2}=1
$$

This quaternion substitution transforms the kinematics equations (eq. 2) to a linear form,

$$
\left[\begin{array}{l}
\dot{a} \\
\dot{b} \\
\dot{c} \\
\dot{d}
\end{array}\right]=\frac{1}{2}\left[\begin{array}{cccc}
0 & r & -q & p \\
-r & 0 & p & q \\
q & -p & 0 & r \\
-p & -q & -r & 0
\end{array}\right]\left[\begin{array}{l}
a \\
b \\
c \\
d
\end{array}\right] \Rightarrow \dot{q}(t)=\omega(p, q, r) q(t)
$$

and the direction cosine matrix becomes

$$
M\left[\begin{array}{l}
\theta \\
\phi \\
\psi
\end{array}\right]=\left[\begin{array}{ccc}
a^{2}+d^{2}-b^{2}-c^{2} & 2(a b+c d) & 2(a c-b d) \\
2(a b-c d) & b^{2}+d^{2}-a^{2}-c^{2} & 2(b c+a d) \\
2(a c+b d) & 2(b c-a d) & c^{2}+d^{2}-a^{2}-b^{2}
\end{array}\right]
$$

The transformation from quaternions to Euler angles is

$$
\begin{aligned}
\sin \theta & =2(b d-a c) \\
\tan \psi & =\frac{2(a b+c d)}{2\left(a^{2}+d^{2}\right)-1} \\
\tan \phi & =\frac{2(b c+a d)}{2\left(c^{2}+d^{2}\right)-1}
\end{aligned}
$$

\section{Solution of the Integrating Equation}

Equation 4 is solved by the integrating factor ${ }^{9}$ method:

$$
Q_{x}(t)=e \int_{t_{0}}^{t} \eta(p, q, r) \mathrm{dt} Q_{x}\left(t_{0}\right)
$$

The integrating factor is

$$
\begin{aligned}
& e \int_{t_{0}}^{t} \eta(p, q, r) \mathrm{dt}=e \int_{t_{0}}^{t} \frac{1}{2}\left[\begin{array}{cccc}
0 & r & -q & p \\
-r & 0 & p & q \\
q & -p & 0 & r \\
-p & -q & -r & 0
\end{array}\right] \mathrm{dt} \\
& =e\left[\begin{array}{cccc}
0 & I_{r} / 2 & -I_{q} / 2 & I_{p} / 2 \\
-I_{r} / 2 & 0 & I_{p} / 2 & I_{q} / 2 \\
I_{q} / 2 & -I_{p} / 2 & 0 & I_{r} / 2 \\
-I_{p} / 2 & -I_{q} / 2 & -I_{r} / 2 & 0
\end{array}\right] \equiv e^{\Omega(p, q, r, t)}
\end{aligned}
$$

where

$$
\frac{I_{r}}{2} \equiv \int_{t_{0}}^{t} \frac{r(t) \mathrm{dt}}{2}, \quad \frac{I_{q}}{2} \equiv \int_{t_{0}}^{t} \frac{q(t) \mathrm{dt}}{2}, \quad \frac{I_{p}}{2} \equiv \int_{t_{0}}^{t} \frac{p(t) \mathrm{dt}}{2}
$$

and can be written in closed form by expanding the exponential in a Taylor series ${ }^{9}$

$$
e^{\Omega}=I+\Omega+\frac{\Omega^{2}}{2}+\frac{\Omega^{3}}{3 !}+\ldots
$$

and noting that

$$
\Omega^{2}=-\left[\frac{I_{p}{ }^{2}+I_{q}{ }^{2}+I_{r}{ }^{2}}{4}\right] \equiv-\left(\frac{\|\omega\|}{2}\right)^{2} I
$$


The matrix, $I$, is the identity matrix. Collecting terms and simplifying gives

$$
e^{\Omega}=\cos \frac{\|\omega\|}{2} I+\frac{2}{\|\omega\|} \sin \frac{\|\omega\|}{2} \Omega \equiv \Phi\left(t, t_{0}\right)
$$

resulting in the homogeneous linear equation

$$
Q_{x}(t)=\Phi\left(t, t_{0}\right) Q_{x}\left(t_{0}\right)
$$

The integrator is made recursive over a small time step, $\Delta t$, using angular rates averaged over the interval

$$
Q_{x_{k+1}}=\Phi_{k+1, k} Q_{x_{k}}
$$

Equation 14 is the "one-step prediction equation," and is always norm preserving. That is, the quaternion magnitude will always be equal to unity, and one does not have to re-normalize the quaternion values after each integration cycle. This ensures greater numerical accuracy and is a unique result not published in navigation literature.

\section{Correction of Integrated Data}

At the end of each integration frame, the algorithm corrects for drift instabilities by using the differences between the magnetic-field and airspeed vectors, measured in body axes and the magnetic-field datum and inertial velocity vector, measured in Earth-relative axes. A particular state error correction is performed only when a fresh measurement is available. To allow for this asynchronous operation, the correction step is partitioned into two parts: a magnetometer correction, and a velocity correction.

The magnetometer correction is performed first. In this step, differences between the observed and expected magnetic-field vector are fed back to stabilize the openloop integration,

$$
\left[\begin{array}{l}
\hat{\theta} \\
\phi \\
\psi
\end{array}\right]_{k+1 / k+1}=\left[\begin{array}{l}
\theta \\
\phi \\
\psi
\end{array}\right]_{k+1 / k}+\kappa_{k+1}\left[Z_{k+1}-M\left[\begin{array}{l}
\theta \\
\phi \\
\psi
\end{array}\right]_{k+1 / k} T_{k+1}\right](15 \mathrm{a})
$$

Here, the matrix $\kappa_{k+1}$ is a "Kalman-style" gain matrix, $T_{k+1}$ is the magnetic-field reference datum in Earth coordinates, and $Z_{k+1}$ is a three-axis magnetometer measurement in body axis.

The vector

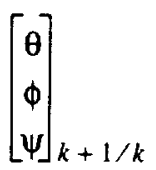

is the predicted state estimate based on open-loop integration over one integration cycle, and the vector

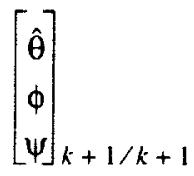

is the state estimate resulting from the magnetic-field vector correction. The matrix $M[$.$] is the transformation$ matrix for rotation from Earth-to-body axis based on the predicted state parameters

$$
\left[\begin{array}{c}
z_{\text {long }} \\
z_{\text {lat }} \\
z_{\text {norm }}
\end{array}\right]=M\left[\begin{array}{c}
\theta \\
\phi \\
\psi
\end{array}\right]_{k+1 / k}\left[\begin{array}{c}
T_{n} \\
T_{e} \\
T_{d}
\end{array}\right]
$$

If no fresh magnetic-field data are available, then the magnetometer correction step is ignored.

After the magnetometer update, the result is corrected using newly acquired velocity data (if available):

$$
\begin{aligned}
{\left[\begin{array}{l}
\theta \\
\phi \\
\psi
\end{array}\right]_{k+1 / k+1}=\left[\begin{array}{l}
\hat{\theta} \\
\phi \\
\psi
\end{array}\right]_{k+1 / k+1} } \\
+K_{k+1}\left[U_{k+1}-M\left[\begin{array}{l}
\hat{\theta} \\
\phi \\
\psi
\end{array}\right]_{k+1 / k+1}\left\{V_{k+1}+W_{k+1}\right\}\right]
\end{aligned}
$$

Here, the matrix $K_{k+1}$ is the gain matrix, $U_{k+1}$ is the body-axis airspeed vector, $V_{k+1}$ is the inertial velocity measurement (from the GPS), and $W_{k+1}$ is the vector representing the local atmospheric winds. As with the previous correction, the vector

$$
\left[\begin{array}{l}
\theta \\
\phi \\
\psi
\end{array}\right]_{k+1 / k+1}
$$

is the new state estimate. As with the magnetometer correction step. if fresh velocity data are unavailable, then the velocity correction step is ignored.

With no direct measurement of the vehicle attitudes available, the wind terms in equation 16 are difficult to sense in real time. Conceptually, the wind vector can be estimated by comparing inertial velocities to airdata 
measurements. But because the inertial velocity vector is sensed in the Earth-relative axis system and the airdata velocity vector is sensed in the body-axis, the vehicle attitudes are implicit in the velocity comparison. This implicitness requires that the attitudes and winds are iteratively estimated. In the iterative method, the attitudes would first be estimated assuming no winds. Using these attitude estimates, the winds are estimated from the differences between the inertial and airdata velocity vectors. The attitudes are then reevaluated iteratively using the estimated winds. Two primary difficulties exist with the iterative method. First, such a complex iterative scheme is very difficult to run in real time; and second, because the attitude equations are highly nonlinear, no guarantee exists that the iterative equations will converge. This nonlinearity makes the iterative algorithm unsuitable for flight-critical navigation.

For simplicity, the winds will either be measured by weather balloon or ignored altogether. As described later in the Results and Discussion section, the winds can generally be measured with good steady-state accuracy by rawindsonde weather balloons, ${ }^{10}$ but these data are subject to diurnal (time) and spatial variation errors. Ignoring the winds, or using wind measurements with large errors, will result in biases in the attitude estimates; however, these errors will not affect the stability of the algorithm. The effects of wind shear will be illustrated in the Results and Discussion section using data from the SRA flight tests.

\section{Computation of the Gain Matrixes}

No optimal method exists for selecting the gain for a nonlinear system of equations; however, if the gain is selected to emulate the form of the Kalman gain matrix, ${ }^{11}$ a robust closed-loop algorithm results. The resulting formula for the gain matrixes are as follows:

$$
\begin{aligned}
& \text { Magnetometer Gain: } \\
& \kappa_{k+1}=\left[P_{k+1 / k}\right]\left[\nabla\left[\begin{array}{l}
\theta \\
\phi \\
\psi
\end{array}\right] M T_{k+1}\right]^{T} \\
& \quad \times\left[\left[\begin{array}{l}
\nabla\left[\begin{array}{l}
\theta \\
0 \\
\psi
\end{array}\right] M T_{k+1} \\
\left.\left[\begin{array}{l}
{\left[P_{k+1 / k}\right.}
\end{array}\right]\left[\nabla\left[\begin{array}{l}
\theta \\
0 \\
\psi
\end{array}\right] M T_{k+1}\right]^{T}+G_{Z_{k+1}}\right]-1
\end{array}\right.\right.
\end{aligned}
$$

\section{Velocity Gain:}

$$
\begin{aligned}
& K_{k+1}=\left[\hat{P}_{k+1 / k+1}\right]\left[\nabla\left[\begin{array}{l}
\hat{\theta} \\
\hat{q} \\
\psi
\end{array}\right] M V_{k+1}\right]^{T} \\
& \times\left[\left[\nabla\left[\begin{array}{l}
\hat{\theta} \\
\phi \\
\psi
\end{array}\right] M V_{k+1}\right]\left[\hat{P}_{k+1 / k}\right]\left[\nabla\left[\begin{array}{l}
\hat{\theta} \\
\hat{\phi} \\
\psi
\end{array}\right] M V_{k+1}\right]^{T}+G_{U_{k+1}}\right. \\
& \left.+M\left[\begin{array}{l}
\hat{\theta} \\
\phi \\
\psi
\end{array}\right]_{k+1 / k+1} G_{V_{k+1}} M^{T}\left[\begin{array}{l}
\hat{\theta} \\
\phi \\
\psi
\end{array}\right]_{k+1 / k+1}\right]^{-1}
\end{aligned}
$$

In equations 17 and 18 , the operator [ $]^{\mathrm{T}}$ is the matrix transpose, $P_{k+1 / k}$ is the error covariance of the integrated state estimate, $\hat{P}_{k+1 / k+1}$ is the error covariance of the state estimate after the magnetometer correction, $G_{Z_{k+1}}$ is the error covariance of the magnetometer measurement vector, $G_{U_{k+1}}$ is the error covariance of the airspeed measurement vector, and $G_{V_{k+1}}$ is the error covariance of the inertial velocity measurement vector. The predicted attitude gradient matrixes are

$$
\left[\nabla\left[\begin{array}{l}
\theta \\
\phi \\
\psi
\end{array}\right] T_{k+1}\right]
$$

$$
=\left[\frac{\partial M\left[\begin{array}{l}
\theta \\
\phi \\
\psi
\end{array}\right]_{k+1}}{\partial \theta} \frac{\partial M\left[\begin{array}{l}
\theta \\
\phi \\
\psi
\end{array}\right]}{\partial \phi} T_{k+1} \frac{\partial M\left[\begin{array}{l}
\theta \\
\phi \\
\Psi
\end{array}\right]}{\partial \psi} T_{k+1}\right]
$$

and

$$
\begin{aligned}
& {\left[\nabla\left[\begin{array}{l}
\theta \\
\phi \\
\psi
\end{array}\right]^{M} v_{k+1}\right]}
\end{aligned}
$$

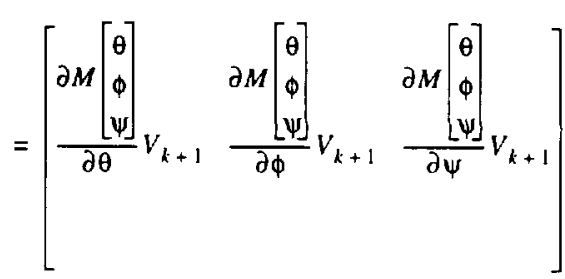


The forms of the gain matrixes determine that very little correction occurs to the integrated state if the measurement (magnetometer and velocity vectors) covariances are large. Conversely, if the integration error covariance is large, then the filter applies a large correction based on the measured data. In this case, the gradient is a sensitivity matrix describing how much correction should be applied to the integrated state vector based on the magnetic-field and velocityvector feedbacks.

\section{Computation of the Enor Covariance Matrices}

A mechanism for evaluating and propagating the error covariances from one data frame to the next must be developed for the filter to be complete. Again, because of the highly nonlinear nature of the problem, an exact expression cannot be developed for the covariance propagations. Instead, approximate expressions are derived from the equations of motion, linearized about the state estimate from the previous data frame.

The error covariance of the integrated state estimate is derived indirectly from the basic kinematics equations. Equation 2 is written here in vector form as

$$
\dot{\Theta}=F[\Theta] \omega
$$

where

$$
\begin{aligned}
\Theta & =\left[\begin{array}{l}
\theta \\
\phi \\
\psi
\end{array}\right], \quad \omega=\left[\begin{array}{l}
p \\
q \\
r
\end{array}\right], \text { and } \\
F[\Theta] & =\frac{1}{\cos \theta}\left[\begin{array}{ccc}
\cos \theta & \sin \phi \sin \theta & \cos \phi \sin \theta \\
0 & \cos \phi \cos \theta & -\sin \phi \cos \theta \\
0 & \sin \phi & \cos \phi
\end{array}\right]
\end{aligned}
$$

By integrating equation 22 over one data frame using explicit $^{12}$ differencing to approximate the time derivative, the result is

$$
\Theta_{k+1 / k}=\Theta_{k / k}+\Delta t\left(F\left[\Theta_{k / k}\right] \omega_{k}\right)
$$

Taking perturbations and collecting terms gives the following error equation:

$$
\begin{aligned}
\delta \Theta_{k+1 / k}=[I & \left.+\Delta t \nabla_{\Theta}\left(F\left[\Theta_{k / k}\right] \omega_{k}\right)\right] \delta \Theta_{k / k} \\
& +\Delta t F\left[\Theta_{k / k}\right] \delta \omega_{k}
\end{aligned}
$$

Defining the covariance matrices

$$
\begin{aligned}
& P_{k+1 / k}=E\left[\delta \Theta_{k+1 / k} \delta \Theta_{k+1 / k}^{T}\right], \\
& P_{k / k}=E\left[\delta \Theta_{k / k} \delta \Theta_{k / k}^{T}\right] \\
& Q_{k}=E\left[\delta \omega_{k} \delta \omega_{k}^{T}\right], \text { etc. }
\end{aligned}
$$

and assuming that the current input error $\delta \omega_{k}$ is uncorrelated with the current state error $\delta \Theta_{k j k}$, then the prediction error covariance update equation can be written approximately as

$$
\begin{aligned}
& P_{k+1 / k} \\
& =\left[I+\Delta t \nabla_{\Theta^{\prime}}\left(F\left[\Theta_{k / k}\right] \omega_{k}\right)\right] P_{k / k}\left[I+\Delta t \nabla_{\Theta}\left(F\left[\Theta_{k / k}\right] \omega_{k}\right)\right]^{T} \\
& +\Delta t^{2} F\left[\Theta_{k / k}\right] Q_{k} F^{T}\left[\Theta_{k / k}\right]
\end{aligned}
$$

After the correction steps are performed, the error covariances of the state estimates are reevaluated in a similar manner by propagating the error through correction equations. The resulting propagation equations are as follows:

\section{magnetometer covariance update:}

$$
\hat{P}_{k+1 / k+1}=\left[I-\hat{K}_{k+1}\left[\nabla\left[\begin{array}{l}
\theta \\
0 \\
\psi
\end{array}\right] T_{k+1}\right] P_{k+1 / k}\right.
$$

velocity covariance update:

$$
P_{k+1 / k+1}=\left[I-K_{k+1}\left[\nabla\left[\begin{array}{c}
\hat{\theta} \\
0 \\
v
\end{array}\right] \quad V_{k+1}\right] \hat{P}_{k+1 / k}\right.
$$

Equations 2, 4, 15, 7, 27, 18, 20, 28, 19, 21, and 29 are the collected algorithm equations. The equation set is repeated recursively until the data stream is exhausted. If at a particular time a new measurement is unavailable, then that correction step is not performed, and the state and covariance matrixes remain unchanged. Figure 4 shows the basic algorithm structure as discussed earlier. 


\section{$\underline{\text { Results and Discussion }}$}

This section presents results that evaluate the performance of the estimating algorithm. The system performance is analyzed for the ultrahigh altitude flight experiment launch trajectory using Monte Carlo error simulations. The estimation algorithm is also demonstrated using flight data derived from the SRA flight tests. Effects of wind shears on the attitude estimate are evaluated using these data.

\section{Six-Degree- of-Freedom Monte Carlo Errors Analyses}

The performance of the attitude estimation algorithm was evaluated by Monte Carlo error simulations generated using the NASA Dryden six-degree-offreedom piloted simulation for the ultrahigh altitude aircraft. Typical balloon-release maneuvers were flown to give representative trajectories for the vehicle launch. These data were then corrupted by bias and random errors, magnetic compass lags, and measurement latencies to simulate the types of real-world measurement errors that would be encountered. Bias errors were introduced into the various measurements at the beginning of each simulation run using a random number generator and a specified bias standard deviation. When the values for the biases were set for a particular data run, they were held constant throughout the remainder of the run. Random errors were generated in a similar manner, except these errors were added to the various data sources at each data frame.

The effects of systematic errors such as compass lags or data latencies were evaluated using various filters that modeled these effects. Also, the piloted launch was repeated several times with different initial launch conditions for heading and initial angular rates. Magnetometer data were generated using a spherical harmonic model of the Earths magnetic field ${ }^{7}$ with the aircraft longitude, latitude, and altitude as inputs to the magnetic-field model. These data were then rotated to body axes and corrupted with bias, random, and compass lag errors. The compass lag errors were added to simulate the induced errors caused by crossing lines of magnetic flux as the aircraft changes pitch attitude or heading attitude. Relative to the expected magnetometer errors, the magnetic-field reference model is extremely accurate, and the resulting reference data are assumed to be known without error.

For each simulation run, the corrupted data were used by the attitude-estimating algorithm to generate Euler angle estimates. Comparisons of the estimated attitude angles with the actual attitude angles (from the simulation) give quantitative measures of the system performance. To obtain ensemble averages of the errors induced by random and bias error components, the simulations were run repeatedly and model output statistics (MOS) were generated. The measurement requirements to achieve the $1.0^{\circ}$ attitude accuracy of the program were determined by this method. Particular attention was paid to determine when a given set of measurements provided closed-loop stability to the algorithm.

Figures 5 through 9 show results of a typical simulation run. Figure 5 shows the basic launch trajectory, where time histories of the airdata parameters (airspeed, angle of attack, altitude, and the roll, pitch, and yaw rates) are presented. Figure 5 shows the uncorrupted and corrupted data that was actually used to perform the analyses. Table 2 presents the bias and random error standard deviations that were used in this simulation run.

The integration is performed at a rate of 25 samples/ $\mathrm{sec}$, the magnetometer data are assumed to be available 5 times/sec, and the velocity data are obtained every 1 $\mathrm{sec}$. The corrupted magnetometer data were lagged using a second-order filter with a time constant of $1.25 \mathrm{sec}$, and a damping ratio of 0.8 .

Figure 6 shows a comparison of the true Euler angles with the attitudes derived from open-loop integration of the corrupted angular-rate data. Clearly, the integration is

Table 2. Bias and random error standard deviations of measurement data.

\begin{tabular}{lll}
\hline \hline Measurement & Bias error & Random error \\
\hline Angular rates, $p, q, r$ & $-0.81,0.72,-0.77 \mathrm{deg} / \mathrm{sec}$ & $\pm 0.25 \mathrm{deg} / \mathrm{sec}$ \\
Magnetic field vector, $Z$ & $-1.7,1.1,0.74$ percent & $\pm 5.0 \%$ \\
Airdata, $V_{\infty}, \alpha, \beta$ & $-2.2 \mathrm{ft} / \mathrm{sec},-0.14^{\circ},-0.15^{\circ}$ & $\pm 5.0 \mathrm{ft} / \mathrm{sec}, \pm 0.25^{\circ}, \pm 0.25^{\circ}$ \\
Inertial velocity, $V_{n}, V_{e}, V_{d}$ & $-1.3,-2.6,-3.7 \mathrm{ft} / \mathrm{sec}$ & $\pm 3.0 \mathrm{ft} / \mathrm{sec}$ \\
Magnetic field datum, $T$ & no error & no error \\
\hline \hline
\end{tabular}




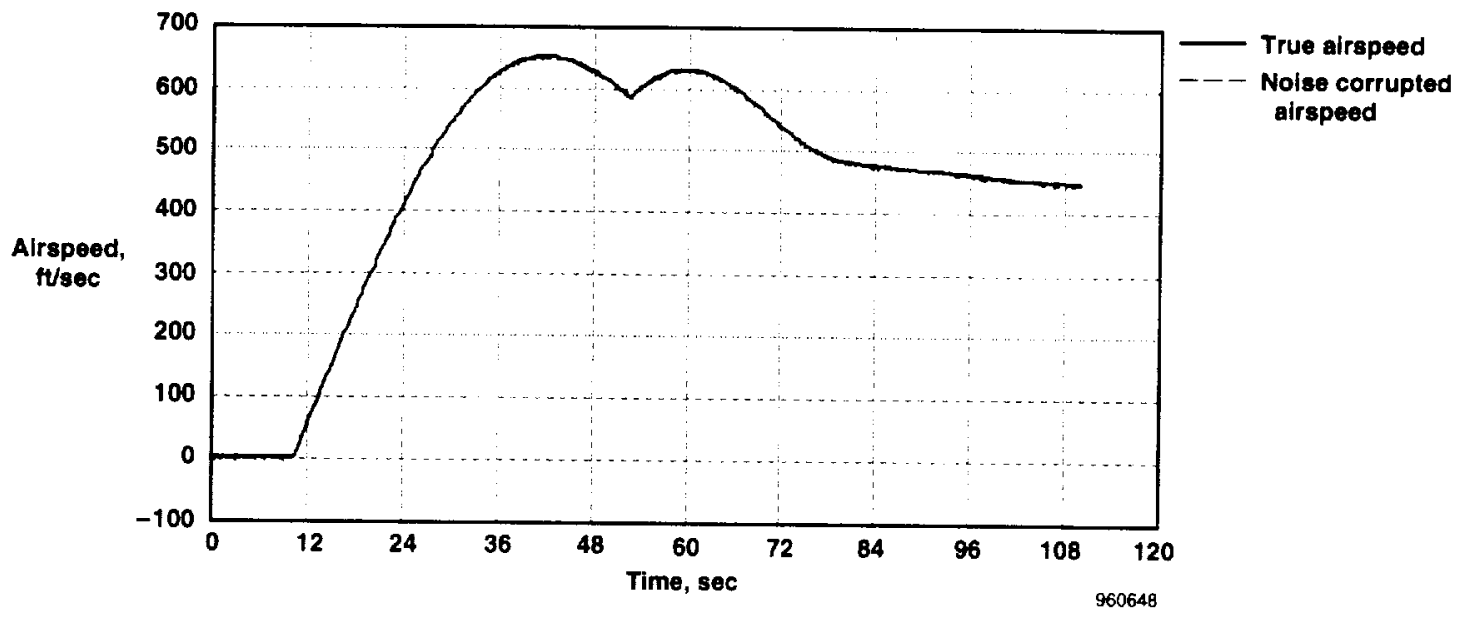

(a) Airspeed.

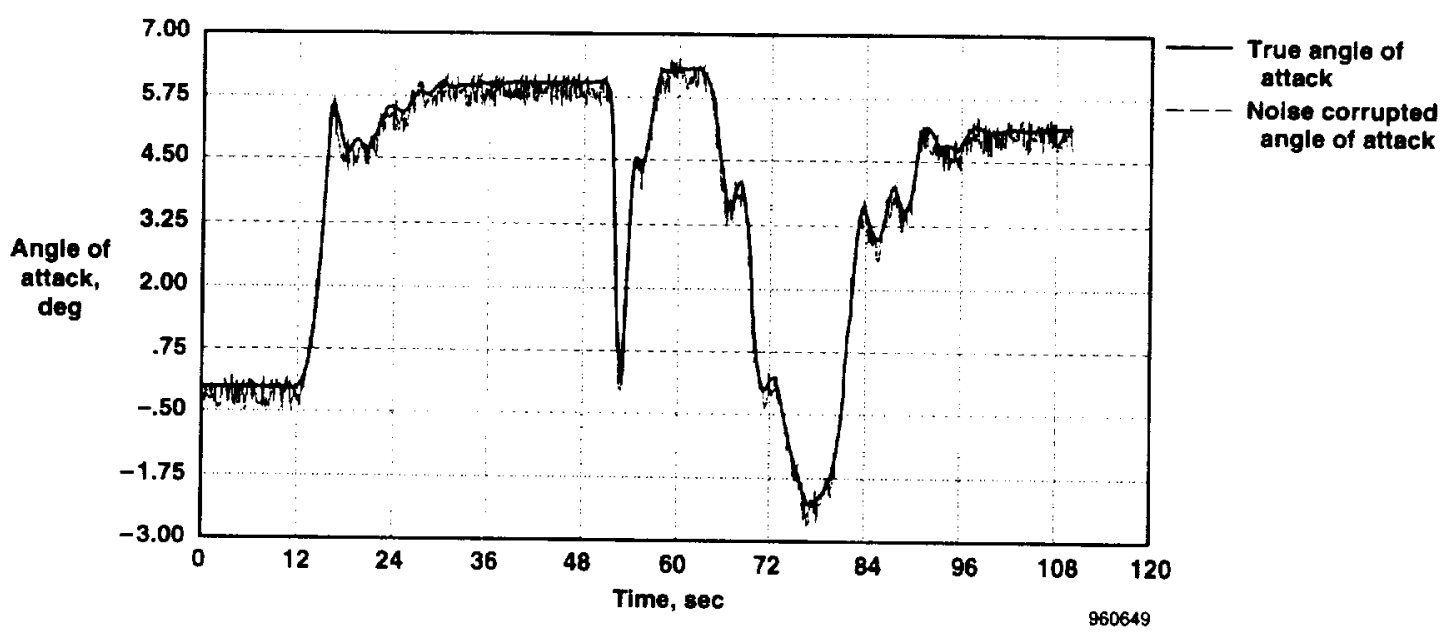

(b) Angle of attack.

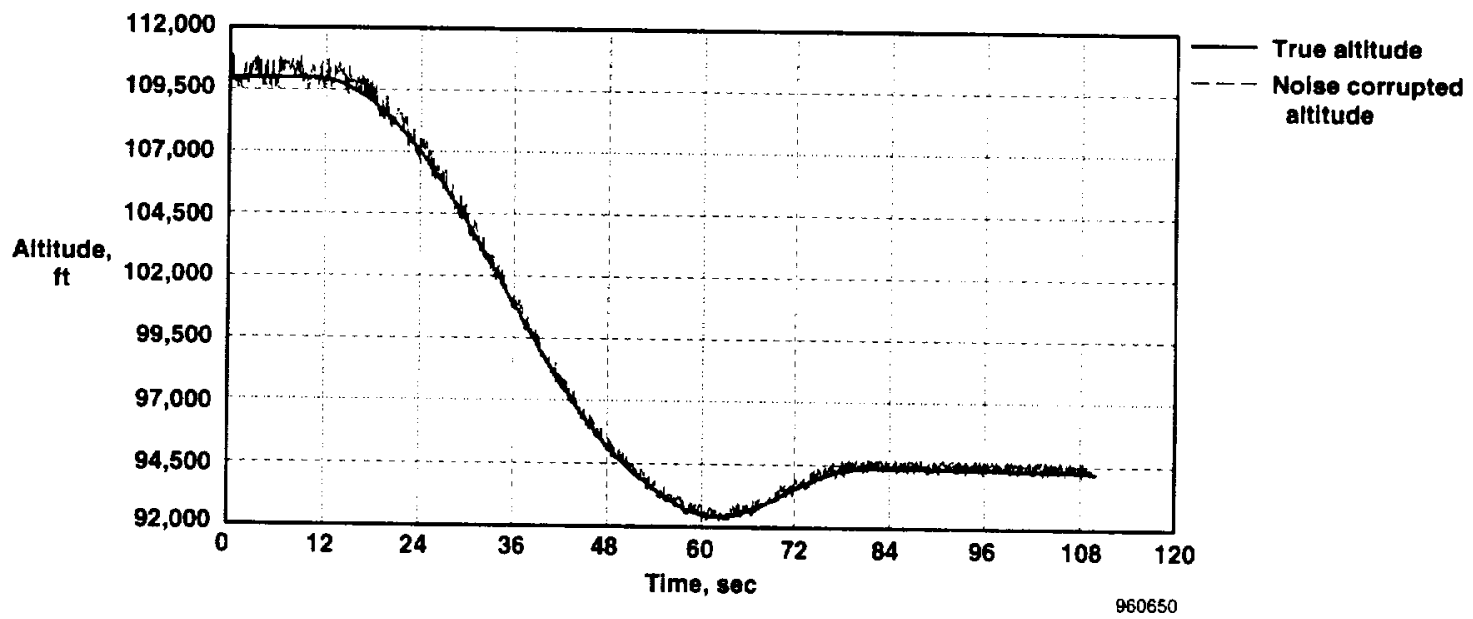

(c) Altitude.

Figure 5. Ultrahigh altitude flight experiment launch trajectory time histories. 


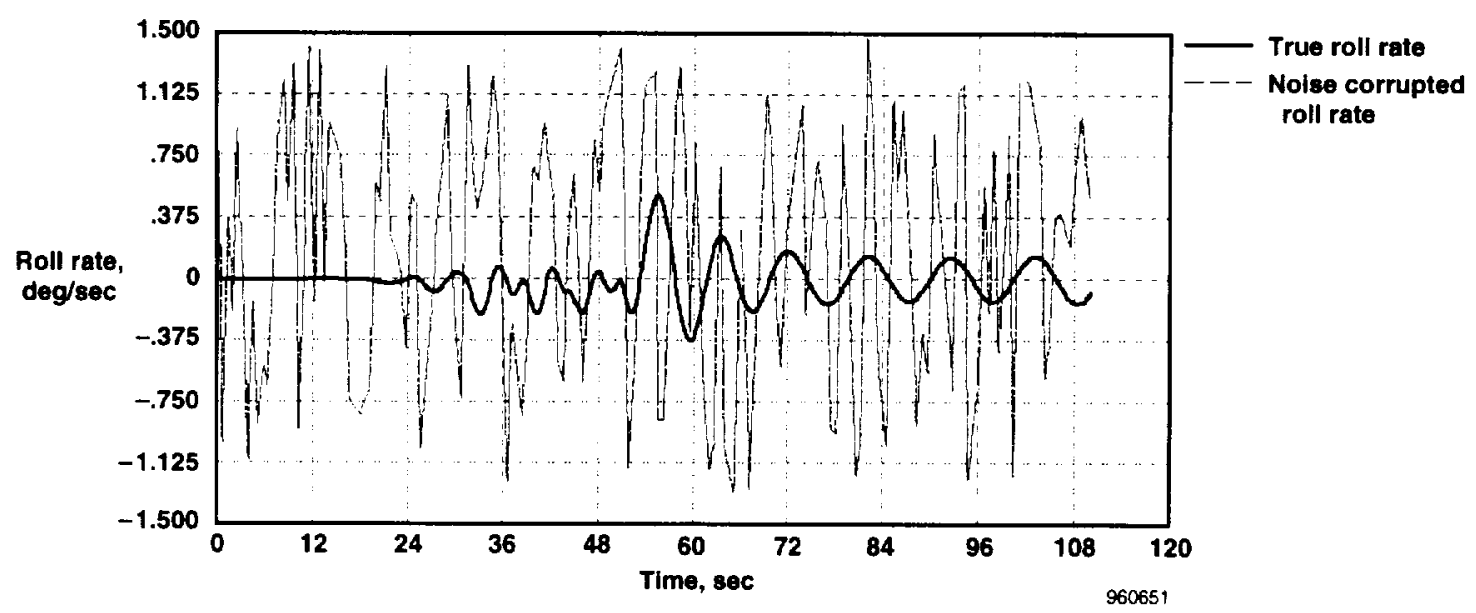

(d) Roll rate.

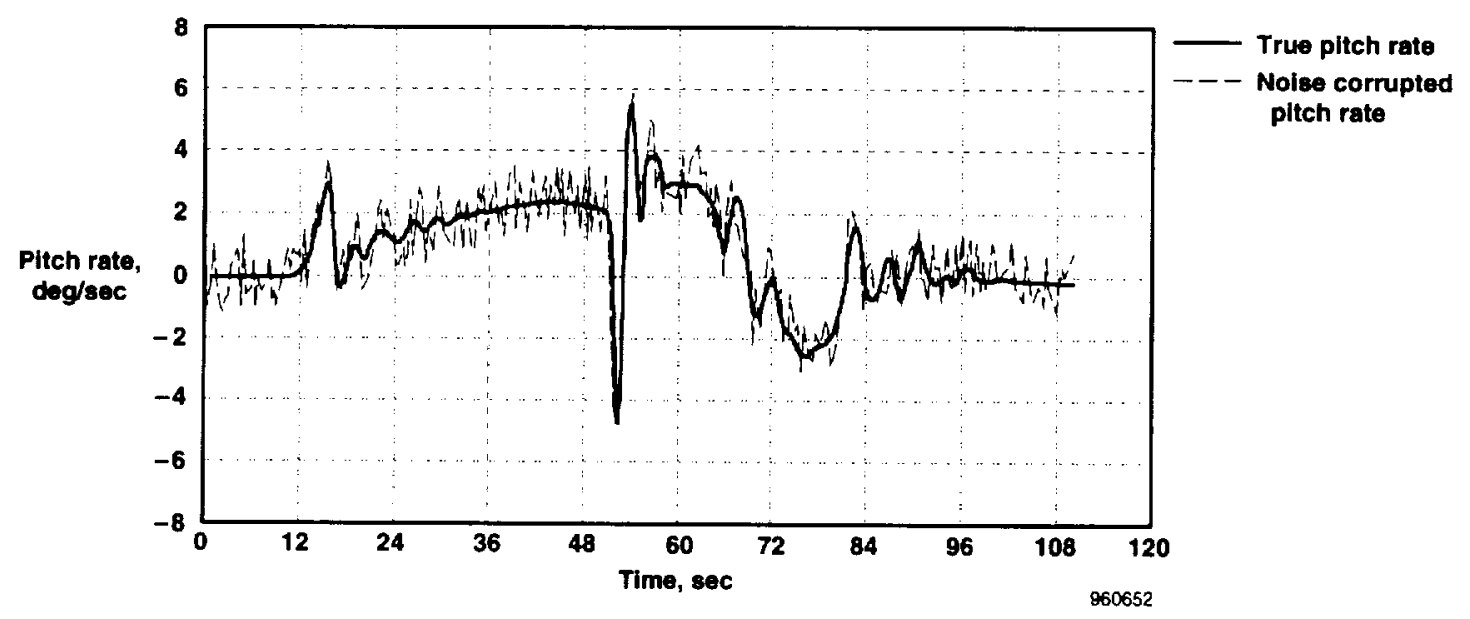

(e) Pitch rate.

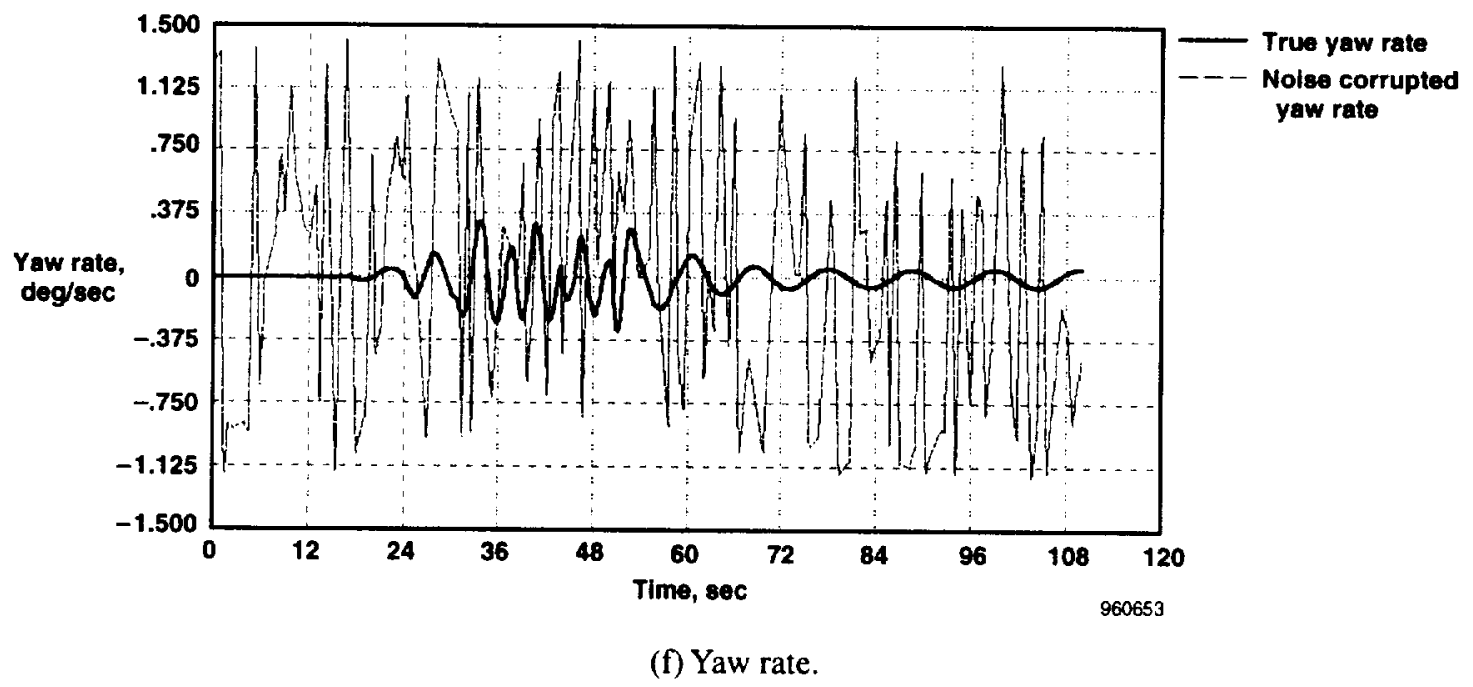

Figure 5. Concluded. 


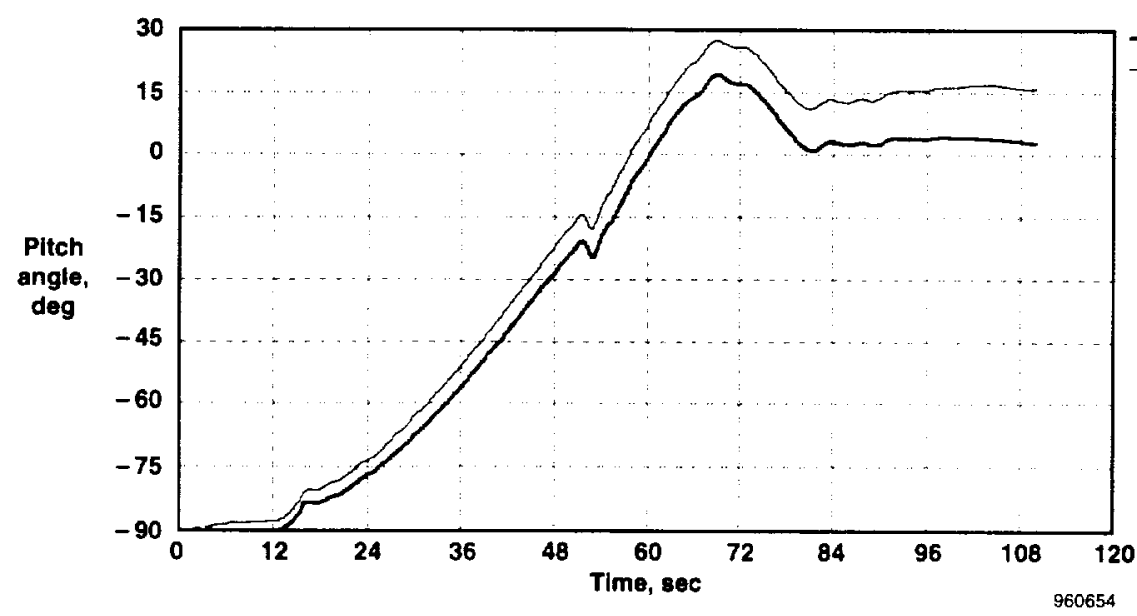

(a) Pitch angle.

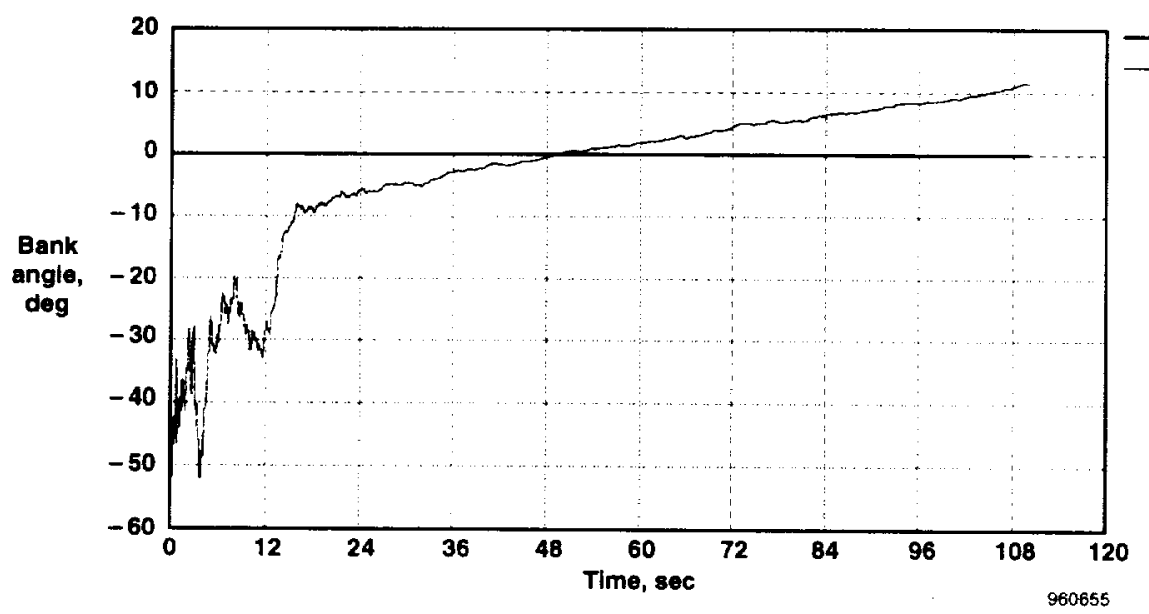

True angle

Open-loop estimate

(b) Bank angle.

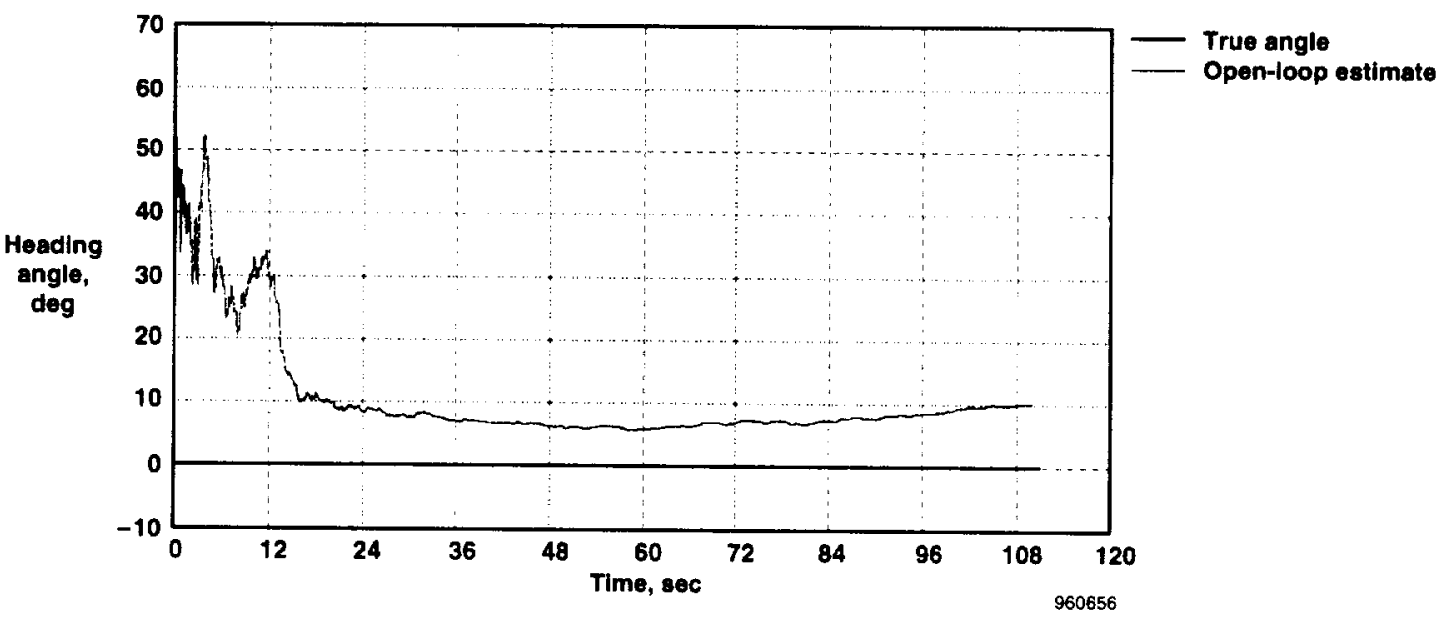

(c) Heading angle.

Figure 6. Launch Euler angle comparisons: true attitudes versus open-loop estimates. 
unstable, and the three attitude angles wander from the true attitudes. Figure 7 shows similar comparisons, except the attitudes have been estimated using the magnetometer data for partial system loop closure. Clearly, the performance is now much better, having only small drifts on pitch and bank angles and a more substantial drift in the heading angle. The system is not yet stable, and the stringent requirements of the ultrahigh altitude flight experiment are not met.

Figure 8 shows the same comparisons, except now only the airspeed and inertial velocity differences have been used to partially close the loop. Again, the system is fairly stable in the pitch and yaw axes, but drifts badly in the roll axis. Because airdata provided only small amounts of information regarding the roll of the vehicle, this result is expected. Figure 9 shows the Euler angle comparisons where magnetometer, airspeed and velocity differences have been used for loop closure. Here, the system loop has been completely closed, and the computations are stable in all three axes. Almost immediately after launch, the estimates converge to the true values. The error equations have been fully stabilized.

Clearly then both the magnetometer and airspeed and inertial velocity stabilizations are required for a completely reliable system. Based on the accuracy and resolution specifications stated earlier, the Monte Carlo analyses MOS were used to assess the maximum bias and random errors allowable to meet the ultrahigh altitude flight program requirements. Table 3 shows estimates of these data for the fully stable system. Measurement latency limitations have yet to be established, but based on the simulation runs, these requirements are very relaxed and can be easily achieved with conventional instrumentation methods.

The key to achieving this instrumentation robustness is in using the two independent attitude references, the magnetic and velocity-vector fields, to completely close the loop on the error equation so that a stable set of (linearized) error equations results. Even in the presence of sizable measurement biases, the system errors will not grow as a function of time.

\section{Evaluation of Attitude-Estimating Algorithm Using Systems Research Aircraft Flight Data}

As mentioned in the introduction, the effectiveness of the estimating algorithm under real flight conditions will be demonstrated using data derived from the SRA flight tests. ${ }^{13}$ The SRA is an F/TF-18 aircraft and is an early model equipped with a high-quality mechanical-gyro INS. ${ }^{14}$ The attitudes from this INS system are used as the reference standard, or "truth model," for this analysis. Estimated accuracies of the INS attitudes are better than $\pm 0.25^{\circ}$ in pitch and bank angle and $\pm 0.5^{\circ}$ in yaw angle. Other outputs from the INS include linear accelerations, angular rates, inertial velocity, flight path and ground track, and aircraft latitude, longitude, and altitude.

The F/TF- 18 is also equipped with an additional set of angular rate sensors that are used as feedback sensors for the flight control system (FCS). ${ }^{14}$ The FCS rate gyros, although efficient dynamically, are subject to small bias errors and systematic drifts. Because FCS rate gyros are independent from the INS (although clearly not as high in quality as the INS angular rate outputs), the FCS data were used for the roll, pitch, and yaw rate inputs to the attitude algorithm. This use of FCS rate gyros avoids any perceived incestuousness in the analysis. Estimated accuracies of the rate data are better than $\pm 0.1 \mathrm{deg} / \mathrm{sec}$ for bias error, and $\pm 0.2 \mathrm{deg} / \mathrm{sec}$ for random error.

Two airdata systems are available on the SRA: the ship system airdata from the airdata computer (ADC), ${ }^{14}$ or the research real-time flush airdata sensing (RT-FADS) system. At low angles of attack, the RT-FADS system and ADC have equivalent levels of accuracy. At greater than $32^{\circ}$ angle of attack, the ADC systems stop operating, whereas the RT-FADS system has been demonstrated to be accurate to a maximum $48^{\circ}$ angle of attack. ${ }^{13}$ For this reason, the RT-FADS airdata were used in this analysis. Estimated accuracies

Table 3. Maximum bias and random errors allowable for the measurement data.

\begin{tabular}{lll}
\hline \hline Measurement & Bias error & Random error \\
\hline Angular rates, $p, q, r$ & $\pm 0.1, \pm 1.50, \pm 2.5 \mathrm{deg} / \mathrm{sec}$ & $\pm 2.0 \mathrm{deg} / \mathrm{sec}$ \\
Magnetic field vector, $Z$ & \pm 10.0 percent & \pm 20 percent \\
Airdata, $U_{\infty}, \alpha, \beta$ & $\pm 10.0 \mathrm{ft} / \mathrm{sec},-0.4^{\circ}, \pm 0.4^{\circ}$ & $\pm 15.0 \mathrm{ft} / \mathrm{sec}, \pm 0.5^{\circ}, \pm 0.5^{\circ}$ \\
Inertial velocity, $V_{n}, V_{e}, V_{d}$ & $\pm 10.0 \mathrm{ft} / \mathrm{sec}$ & $\pm 15.0 \mathrm{ft} / \mathrm{sec}$ \\
\hline \hline
\end{tabular}




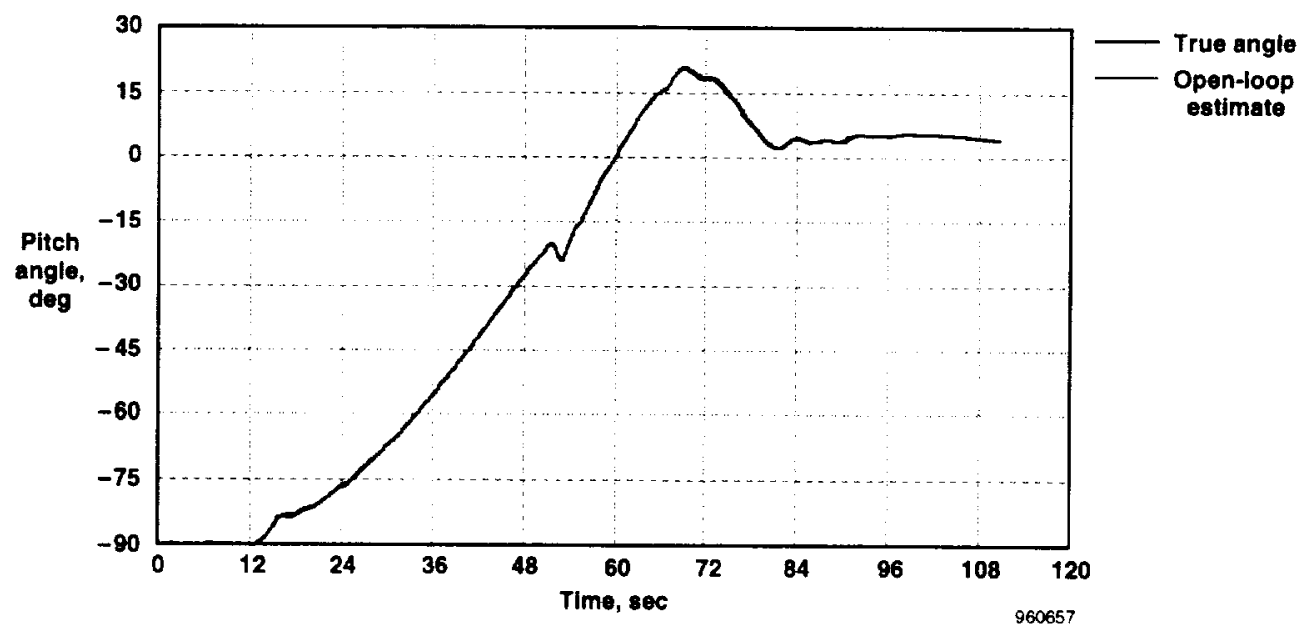

(a) Pitch angle.

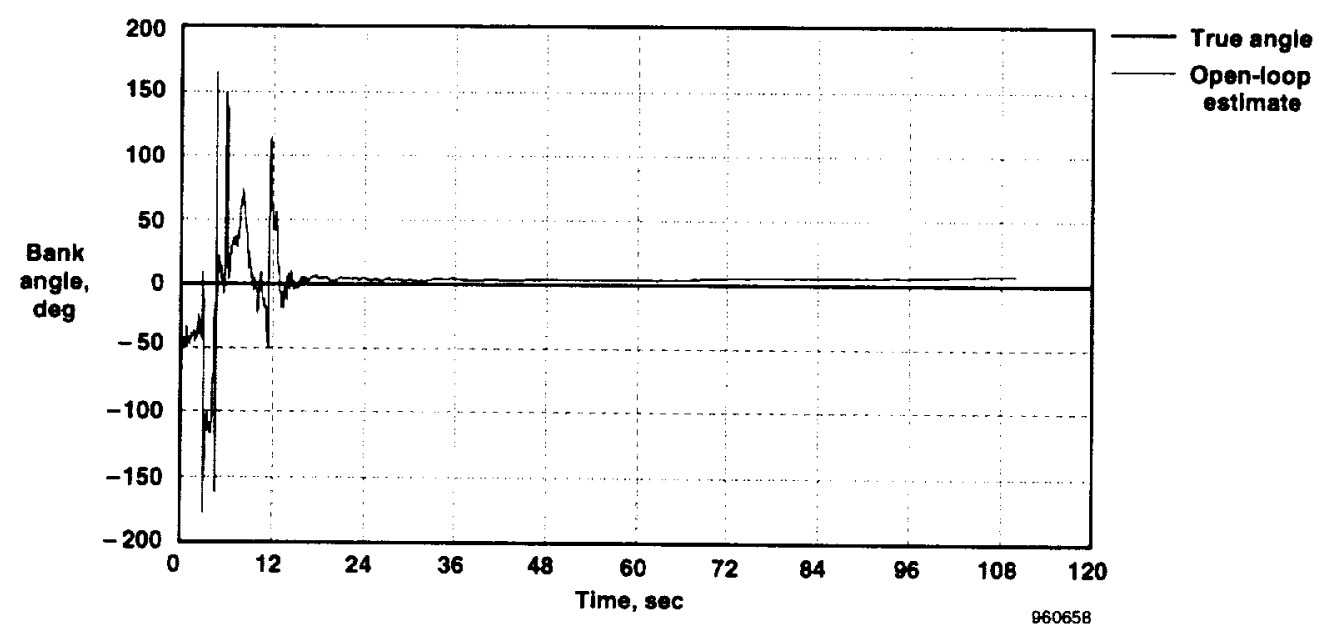

(b) Bank angle.

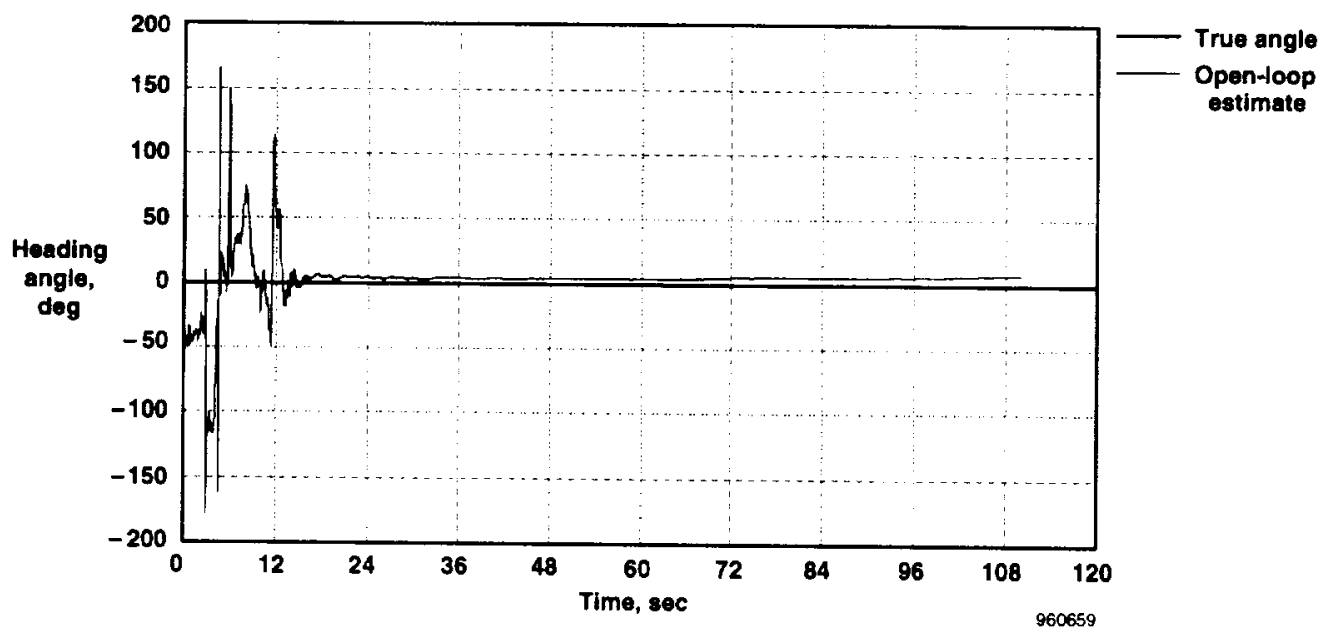

(c) Heading angle.

Figure 7. Launch Euler angle comparisons: true attitudes versus - magnetometer only - estimates. 


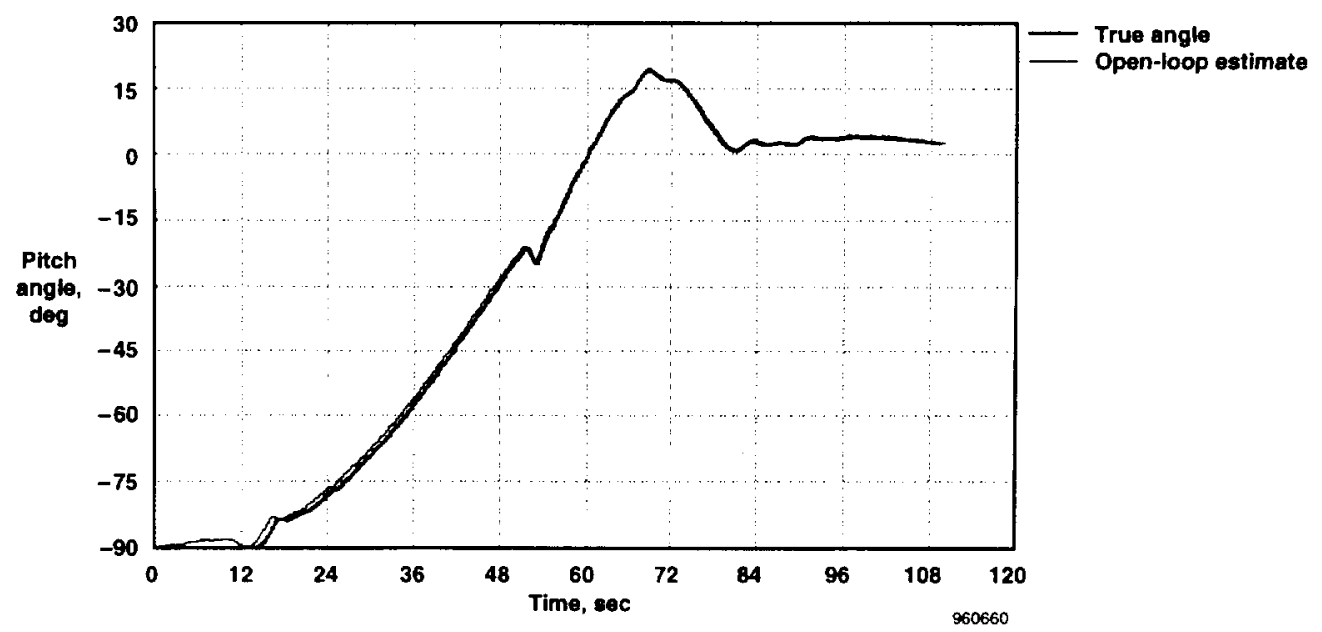

(a) Pitch angle.

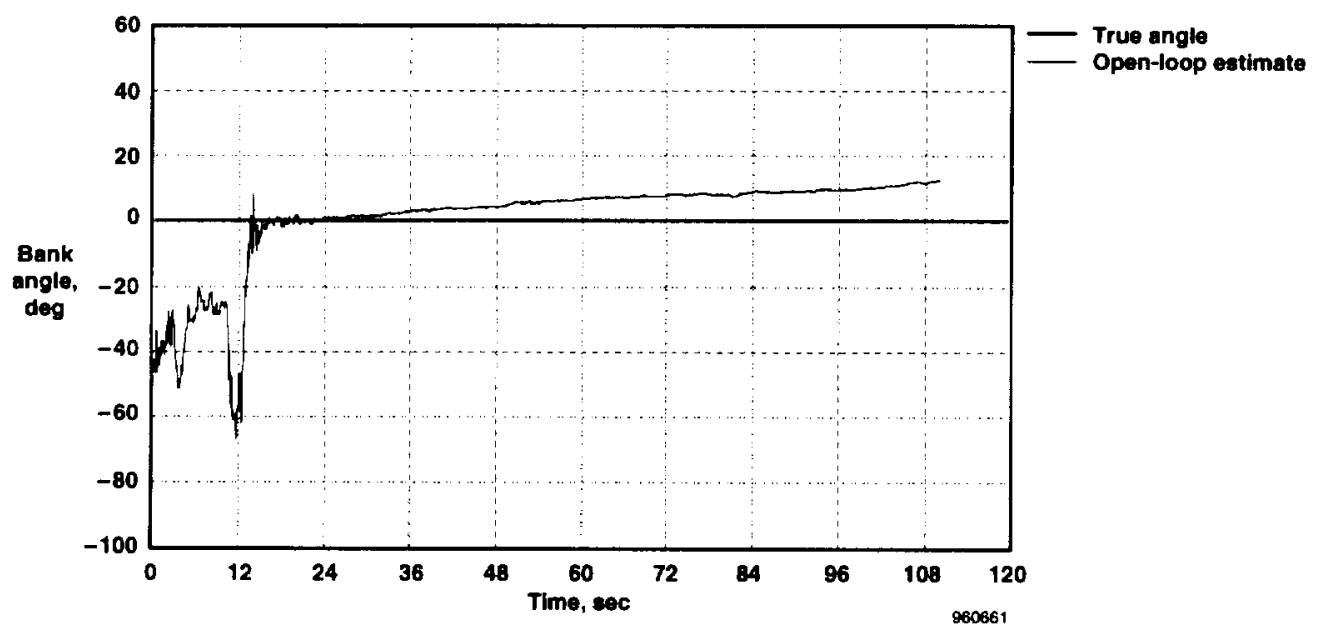

(b) Bank angle.

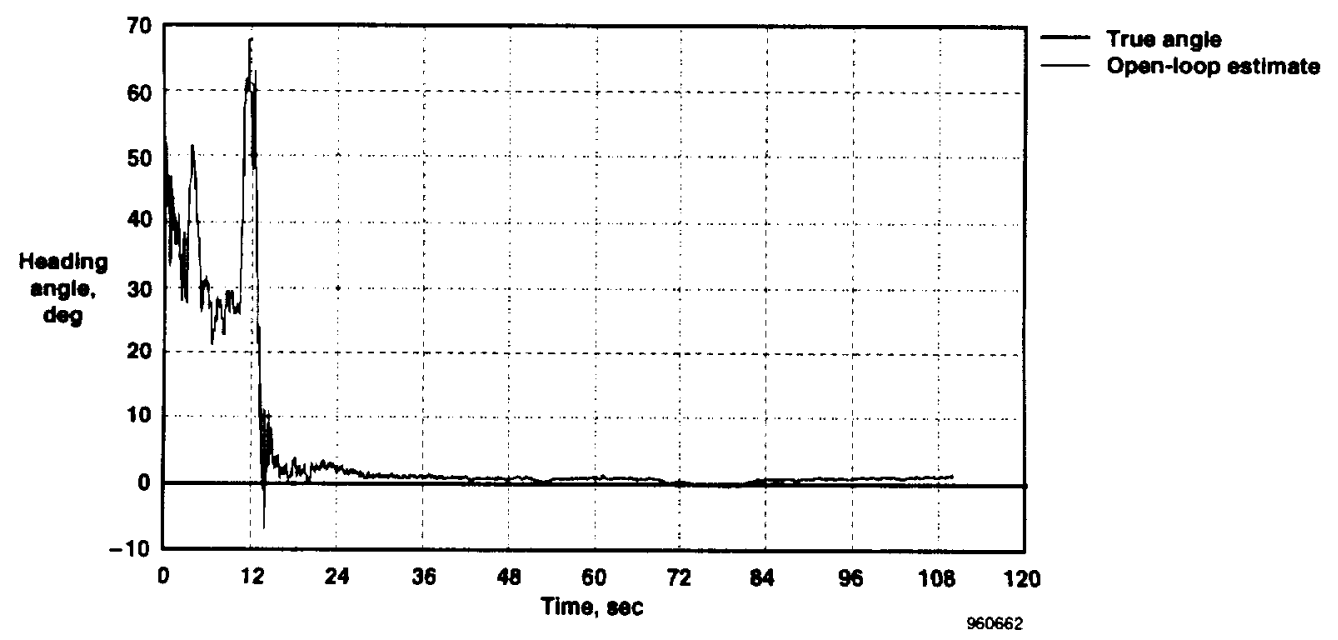

(c) Heading angle.

Figure 8. Launch Euler angle comparisons: true attitudes versus airdata only estimates. 


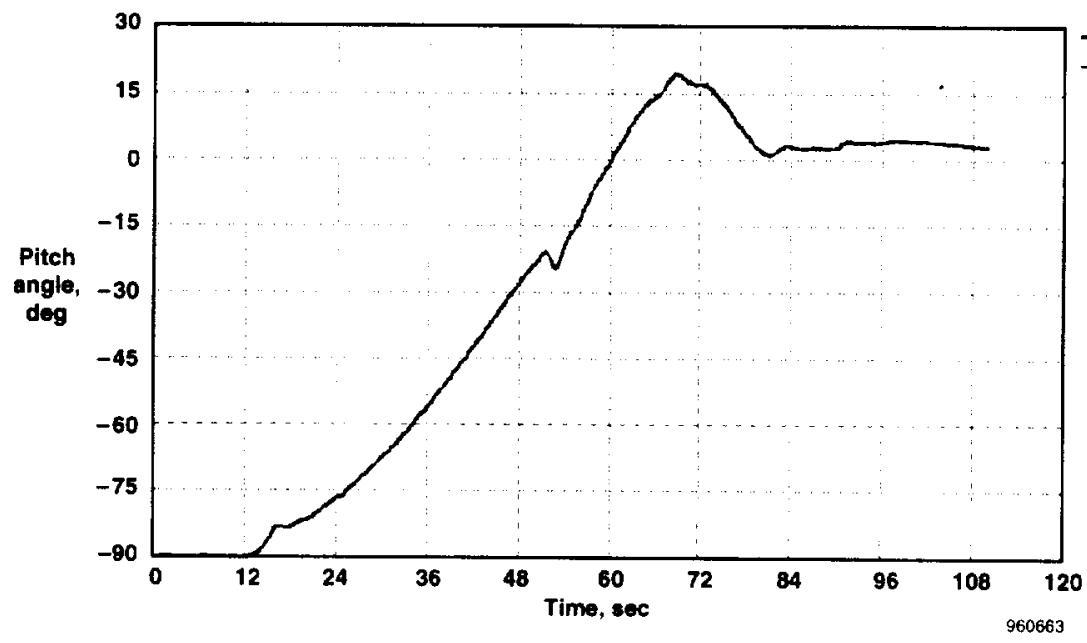

(a) Pitch angle.

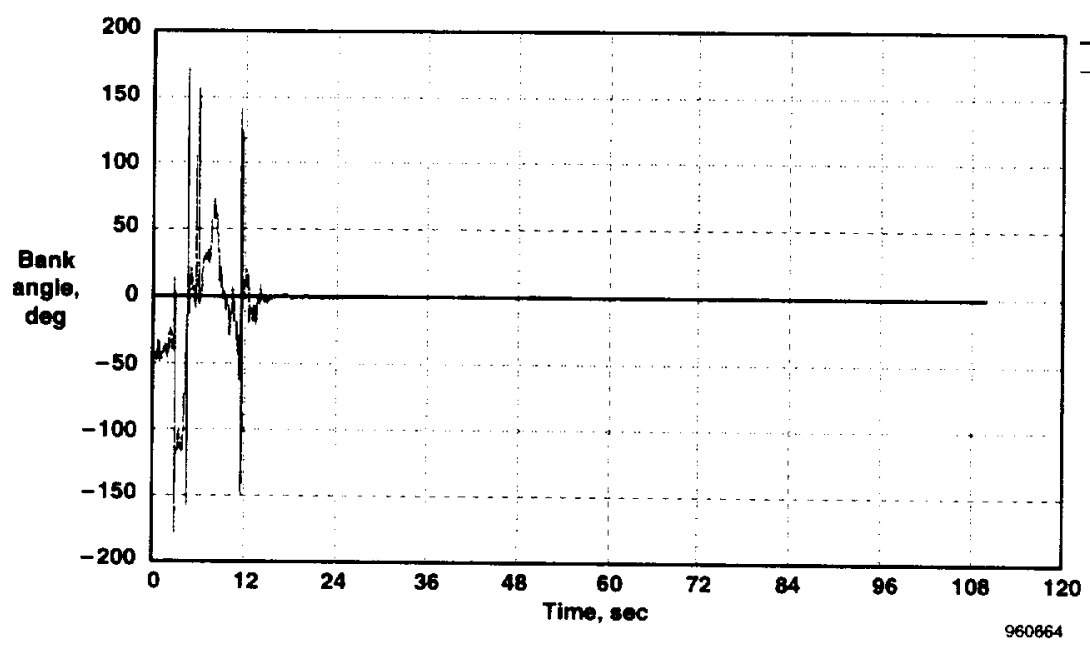

True angle

Open-loop estimate

(b) Bank angle.

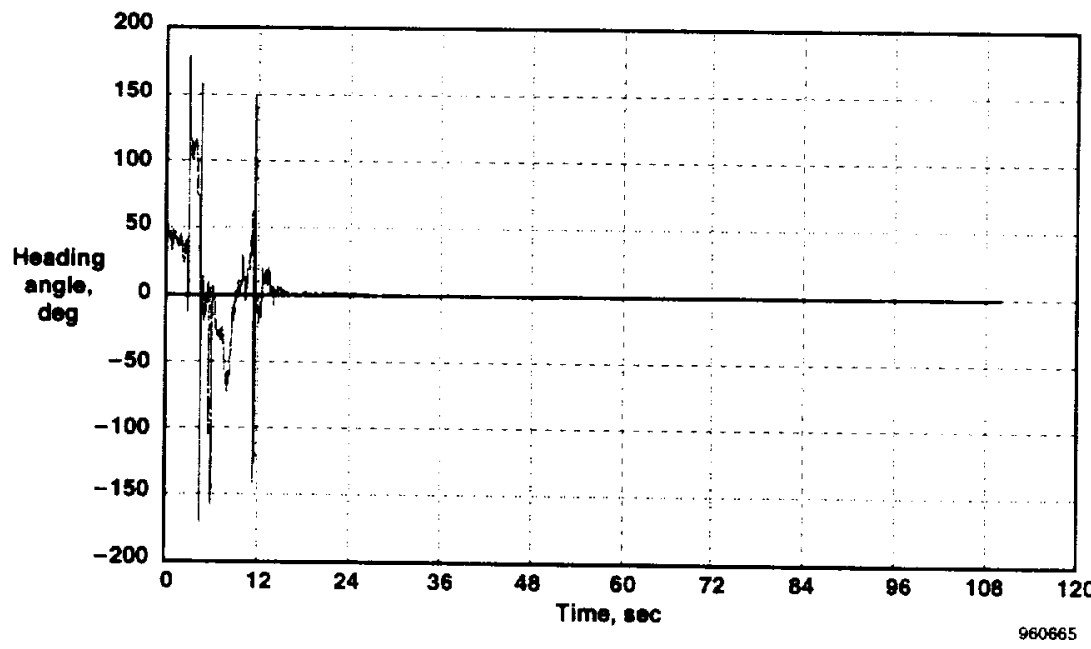

True angle

Open-loop estimate

(c) Heading angle.

Figure 9. Launch Euler angle comparisons: true attitudes versus magnetometer estimates. 
of the RT-FADS system ${ }^{13}$ are 3-5 $\mathrm{ft} / \mathrm{sec}$ in airspeed, and $\pm 0.25^{\circ}$ in angle of attack, and angle of sideslip. The system has been tested and found to be reliable to a maximum of Mach 1.60 .

Again, to avoid any perceived incestuousness in the analysis, the inertial velocity measurements from the INS were not used in this analysis. Because, the SRA was not equipped with a GPS, inertial velocity estimates were obtained using $\mathrm{C}$-band radar tracking data. In this procedure, space-positioning data are numerically differentiated to give velocities relative to the Earth-axis system. For radar elevation angles greater than $10^{\circ}$, the random accuracies in the radar velocity data are believed to be approximately $\pm 4-8 \mathrm{ft} / \mathrm{sec}$ and are of necessity unbiased (because of the numerical differentiation). ${ }^{13}$ Time skews resulting from the differentiating filters were removed before processing the data in the attitude algorithm. Although not truly a strap-down measurement, these C-band data are a good approximation of the type of data that would be acquired by an onboard GPS system.

Three-axis magnetic-field measurements were not available on the SRA vehicle. Instead, the F/TF-18 SRA has a single-axis magnetic compass available as a part of the cockpit instrumentation, and this instrument was used to generate a pseudo 3-axis magnetometer data set. The estimated compass accuracy is $\pm 1^{\circ}$ in bias, and $\pm 5^{\circ}$ random. For a given latitude and longitude, the reference magnetic-field datum vector (from the spherical harmonic model) was rotated about the vertical axis until the vector angle from magnetic north matched the compass heading. Similarly, a pseudo pitch angle was generated from the radar flightpath angle and the angle-of-attack measurement. This angle was used to rotate the magnetic-field vector to the proper pitch attitude. As no data for bank angle were available independent of the INS measurements, the magneticfield vector was not rotated about the roll axis. To prevent erroneous data corrections caused by this lack of roll information, the magnetometer correction was performed only when the absolute value of the predicted bank angle was less than $10^{\circ}$. This lack of bank angle information at high roll angles presents a less-than-ideal scenario for the estimating algorithm.

\section{Flight 540 Results}

Results from SRA flight 540 will now be presented. Calculations are performed from takeoff to landing and are typical of results from other SRA flights. All of the data used are from the sources described in the previous section. Figure 10 shows time histories of the airdata parameters airspeed, angle of attack, and altitude, and the angular-rate parameters roll, pitch and yaw rate.
As with the attitude analyses presented earlier in the paper, figures 11 and 12 show comparisons of the estimated attitudes and INS attitudes. Figure 11 shows a comparison of the INS-derived Euler angles with the attitudes derived from open-loop integration. The rategyro integration was performed at a rate of 25 samples/ sec. Clearly, the open-loop estimates are unstable, and the Euler components wander around the INS values throughout the flight. Figure 12 shows attitude comparisons where corrections have been applied to compass, velocity, and airdata once every second to stabilize the rate-gyro integration. As predicted by the earlier Monte Carlo simulations, the error equation is stabilized and no significant long-term attitude drift occurs.

Figure 13 shows a subset of the data shown in figure 12, but with an expanded time scale to more clearly show the level of agreement between the INS and estimated attitudes. For bank angles less than $10^{\circ}$, the agreements are within the specified requirements for this experiment (errors less than $\pm 0.5^{\circ}$ in pitch axis and $\pm 1.0^{\circ}$ in roll). For roll angles larger than $10^{\circ}$, the estimates degrade in both pitch and roll axes, with pitch angle errors as large as $-3^{\circ}$ and roll angle errors as large as $-10^{\circ}$. As mentioned previously the deviation from INS attitudes is not surprising given that for this analysis no magnetic field correction is applied for bank angles at greater than $10^{\circ}$. The ultrahigh altitude flight experiment heading accuracy requirement of $\pm 5^{\circ}$ is met throughout the entire flight. Analyses of data from other SRA flights have produced similar results.

\section{The Effects of Wind Shear on the Attitude Estimates}

As mentioned earlier, the wind velocity will be typically ignored by the ultrahigh altitude flight experiment algorithm because measuring in real time is difficult. In the presence of significant wind shears, the result of ignoring the winds is a bias or systematic error in the attitude estimates. Figures 14 and 15 show the effect of wind shear using data from the SRA flight 540 . For this flight, the atmospheric winds were measured before and after flight using rawinsonde"l weather balloon soundings, interpolated as a function of time to minimize the effects of diurnal variations in the winds.

During the flight, the vehicle rapidly climbed from an altitude of $10,000 \mathrm{ft}$ to an altitude of $40,000 \mathrm{ft}$, resulting in a large change in the easterly component of the wind. Figure 14 shows time histories of the horizontal wind components and altitude. The full feed back (magneticfield, airdata, and velocity) attitude-estimating algorithm was run for this segment of data with and without the measured winds being included in the computations. 


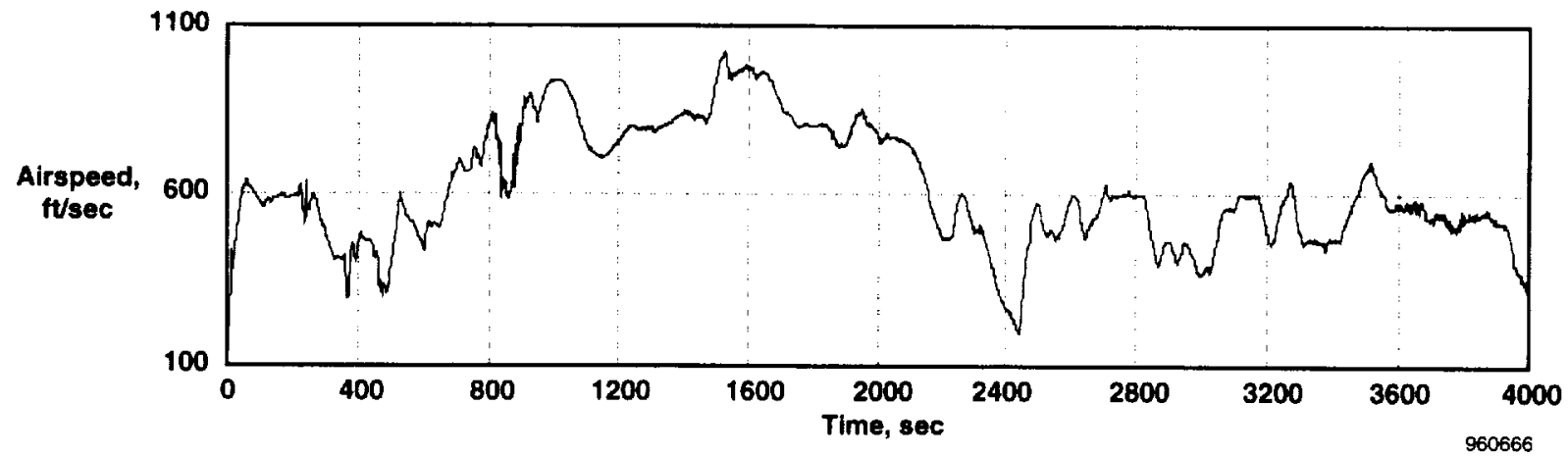

(a) Airspeed.

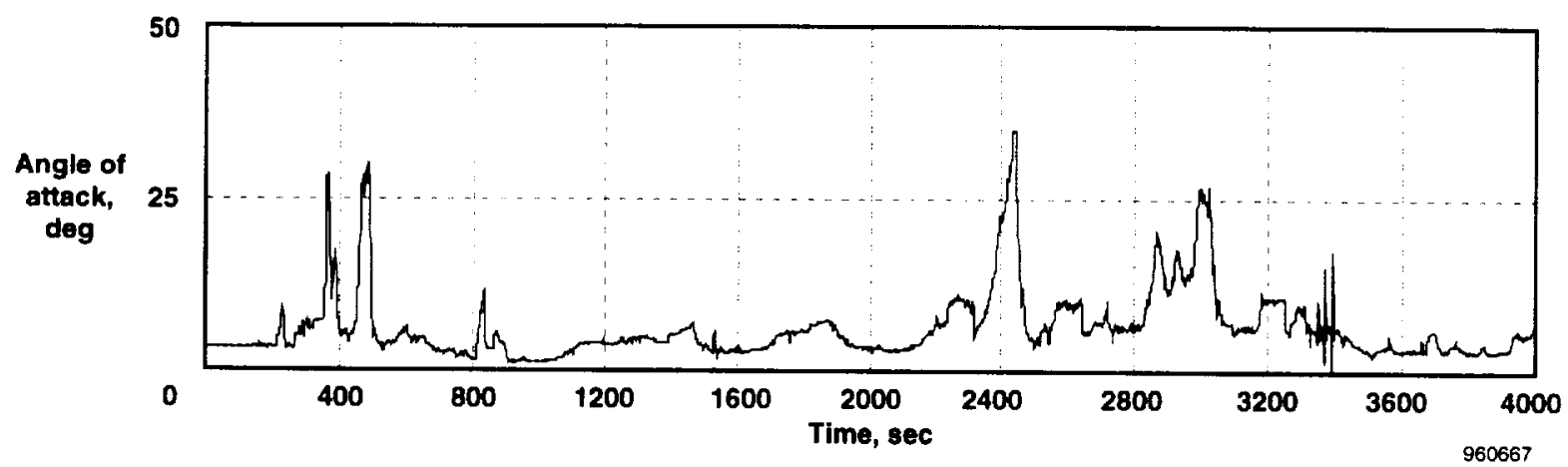

(b) Angle of attack.

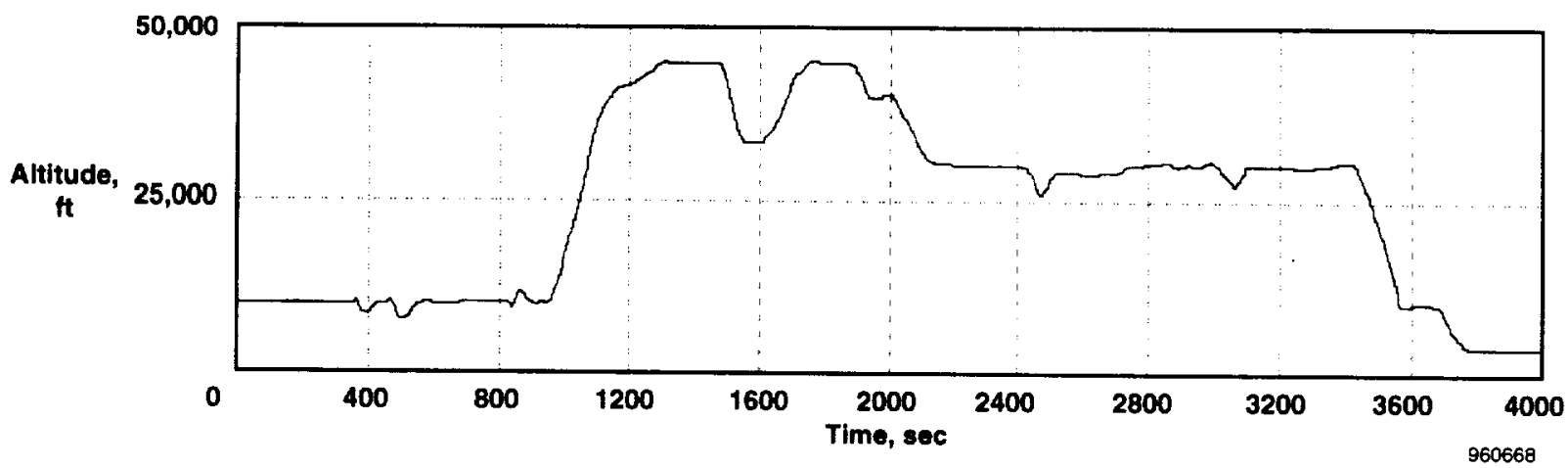

(c) Altitude.

Figure 10. SRA fight 540 time history, takeoff to landing. 


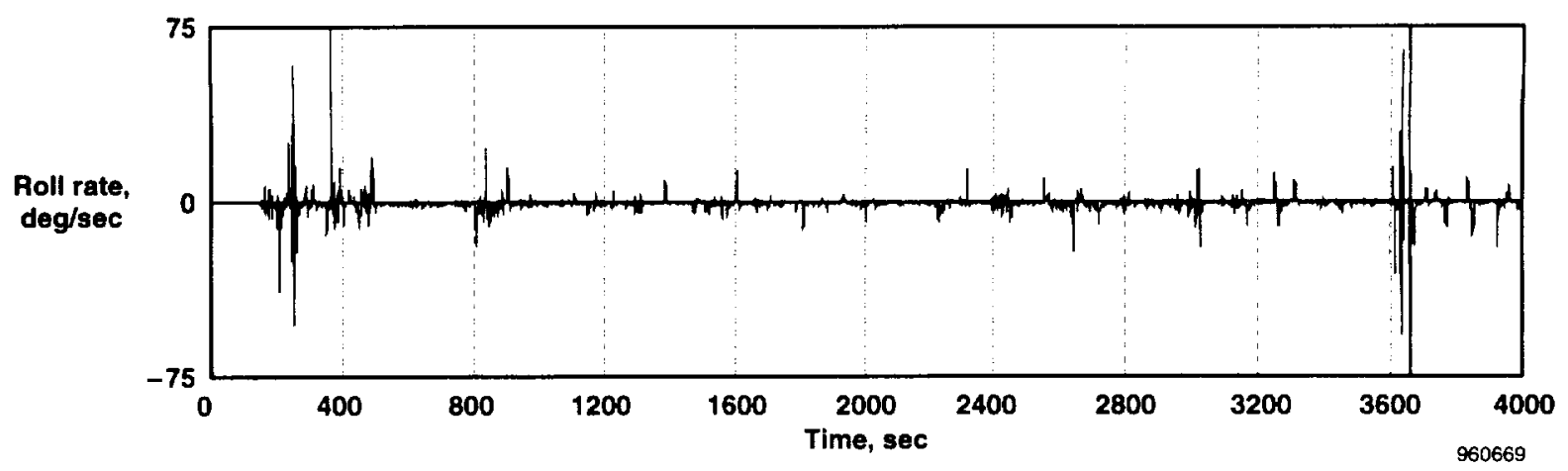

(d) Roll rate.

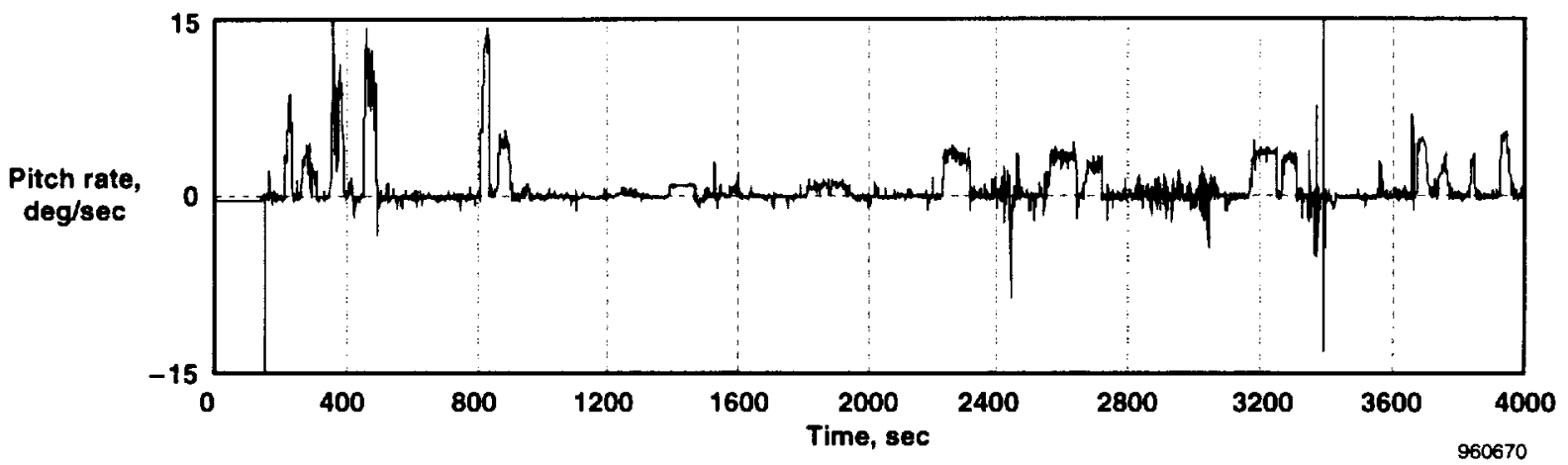

(e) Pitch rate.

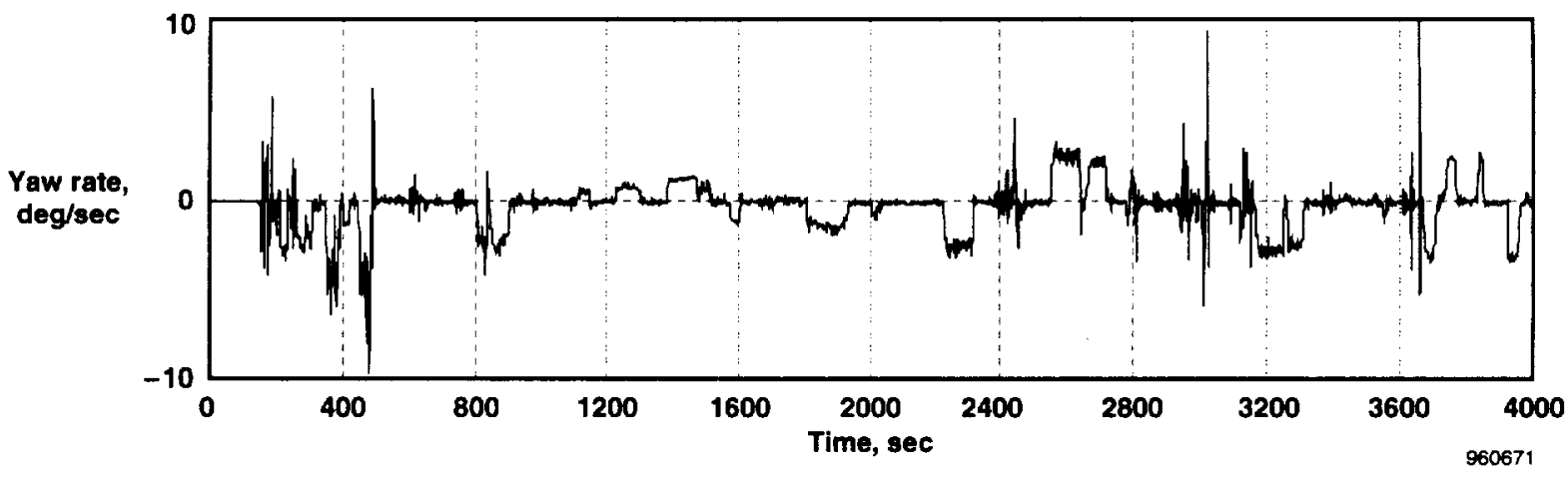

(f) Yaw rate.

Figure 10. Concluded. 


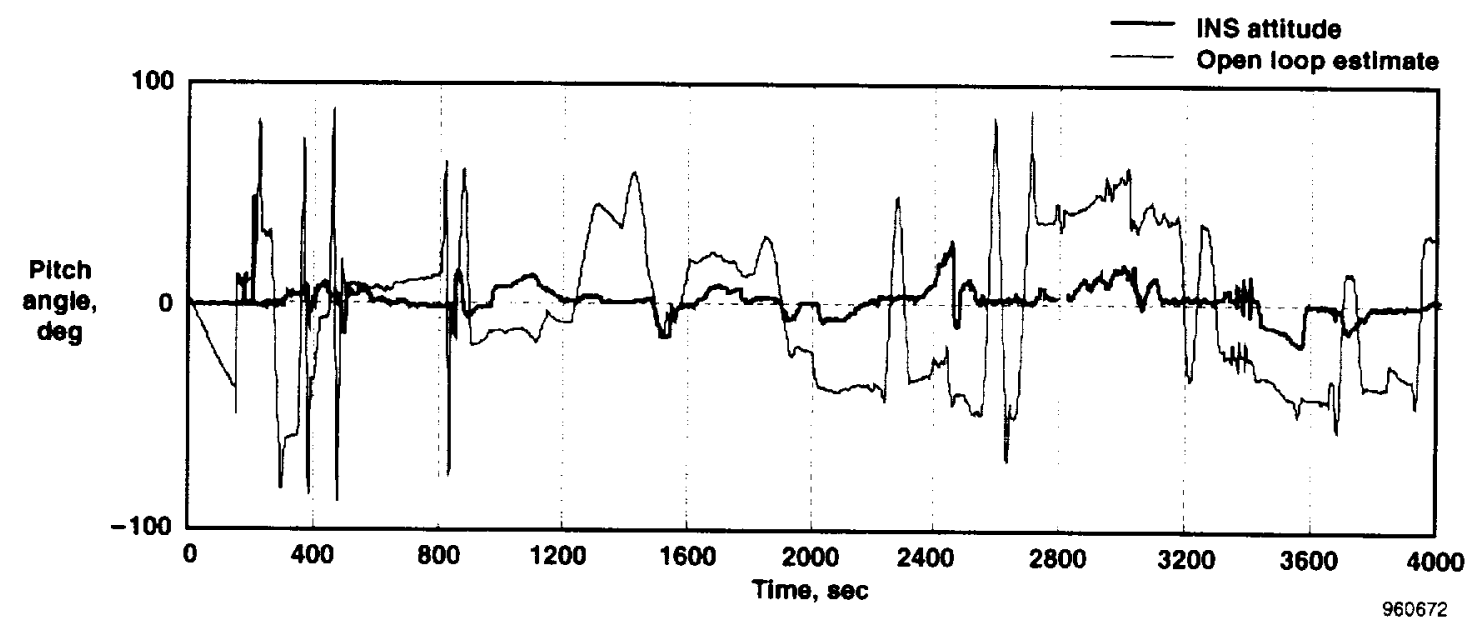

(a) Pitch angle.

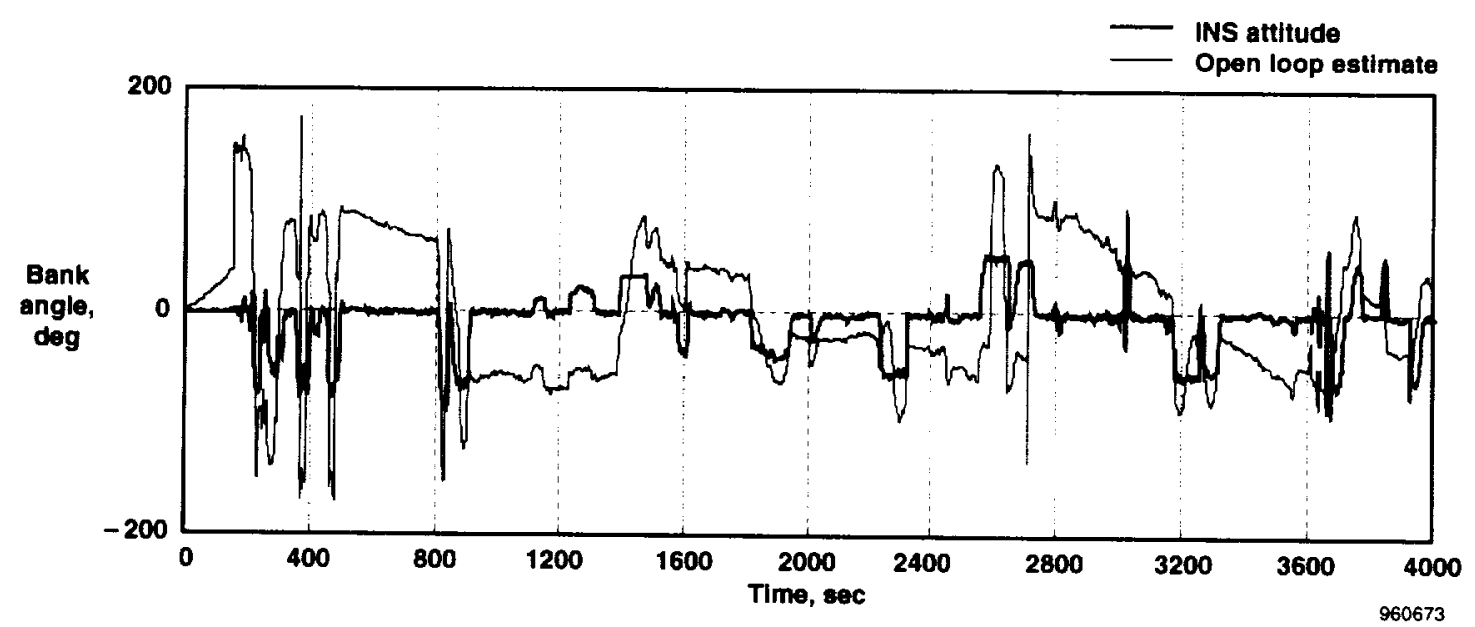

(b) Bank angle.

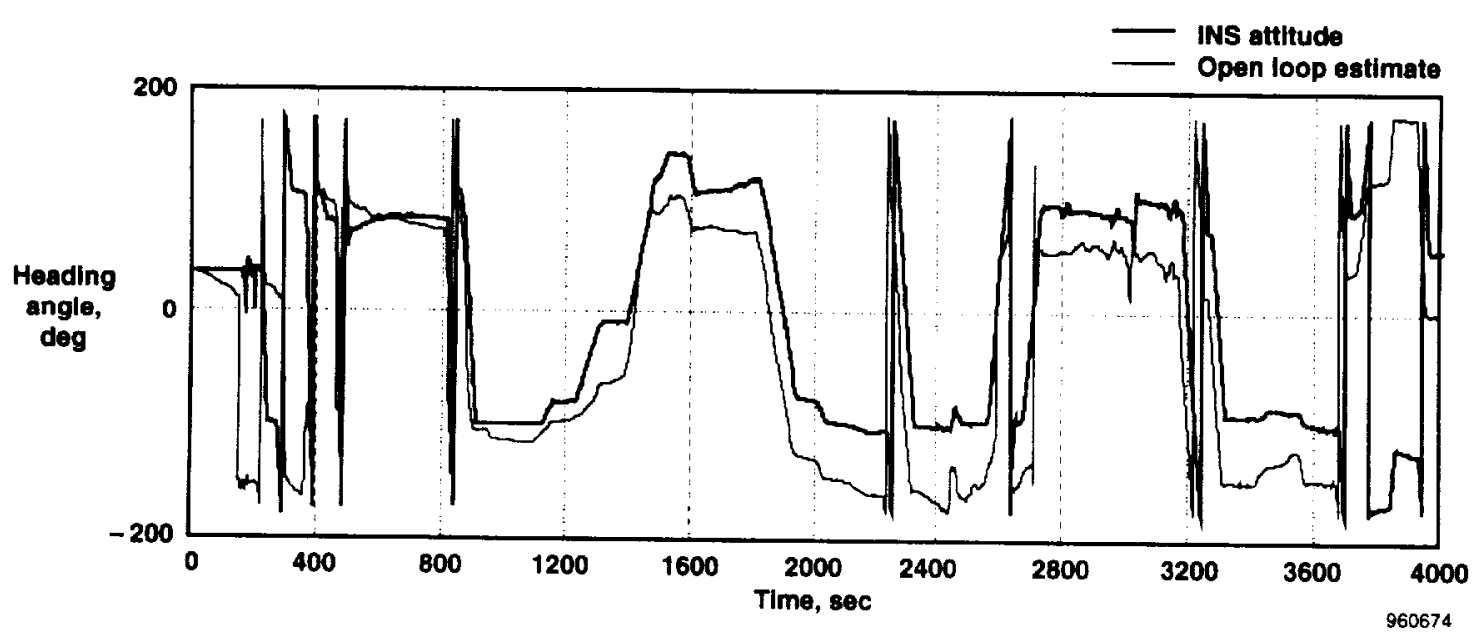

(c) Heading angle.

Figure 11. SRA flight 540 Euler angle comparisons INS to open-loop-estimates. 


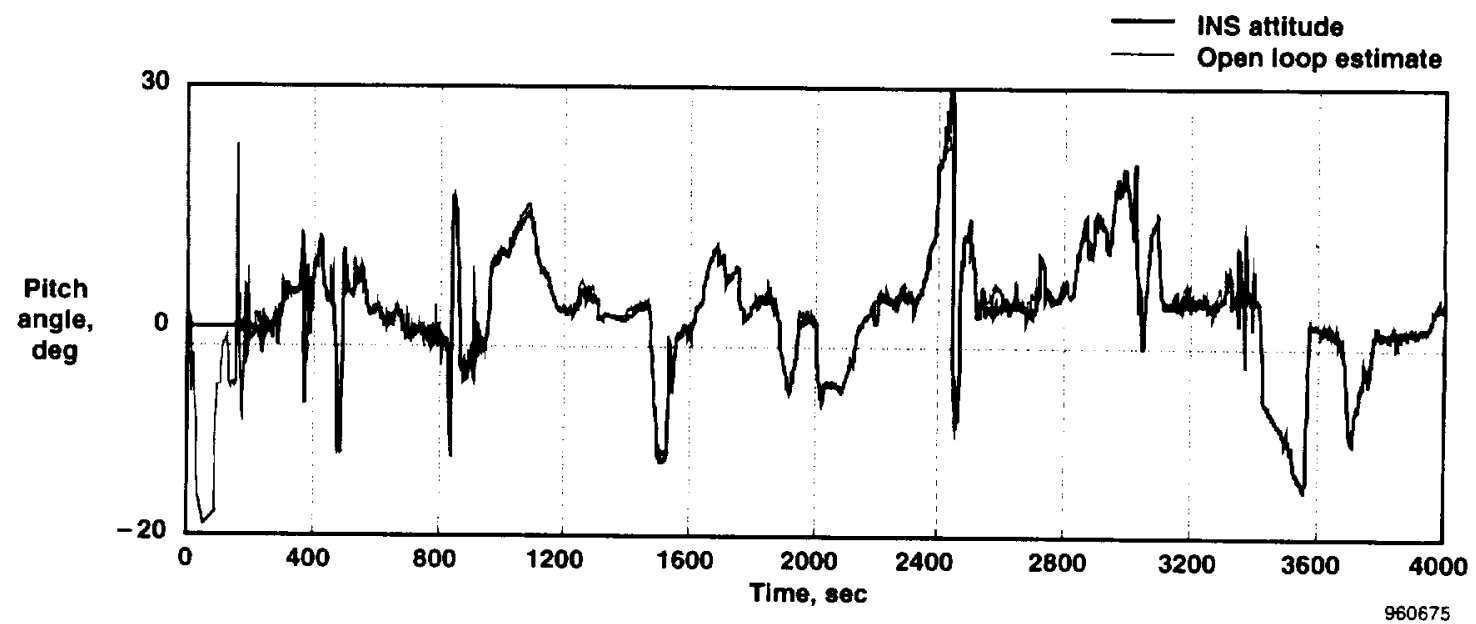

(a) Pitch angle.

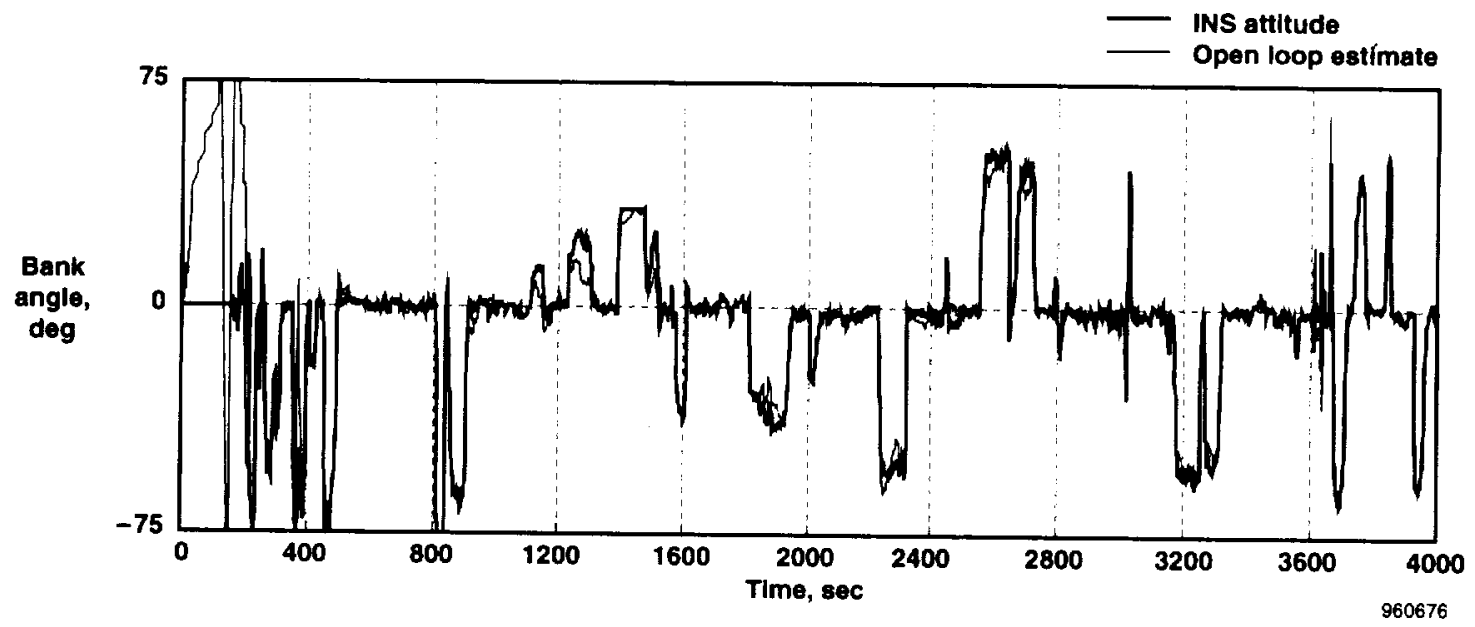

(b) Bank angle.

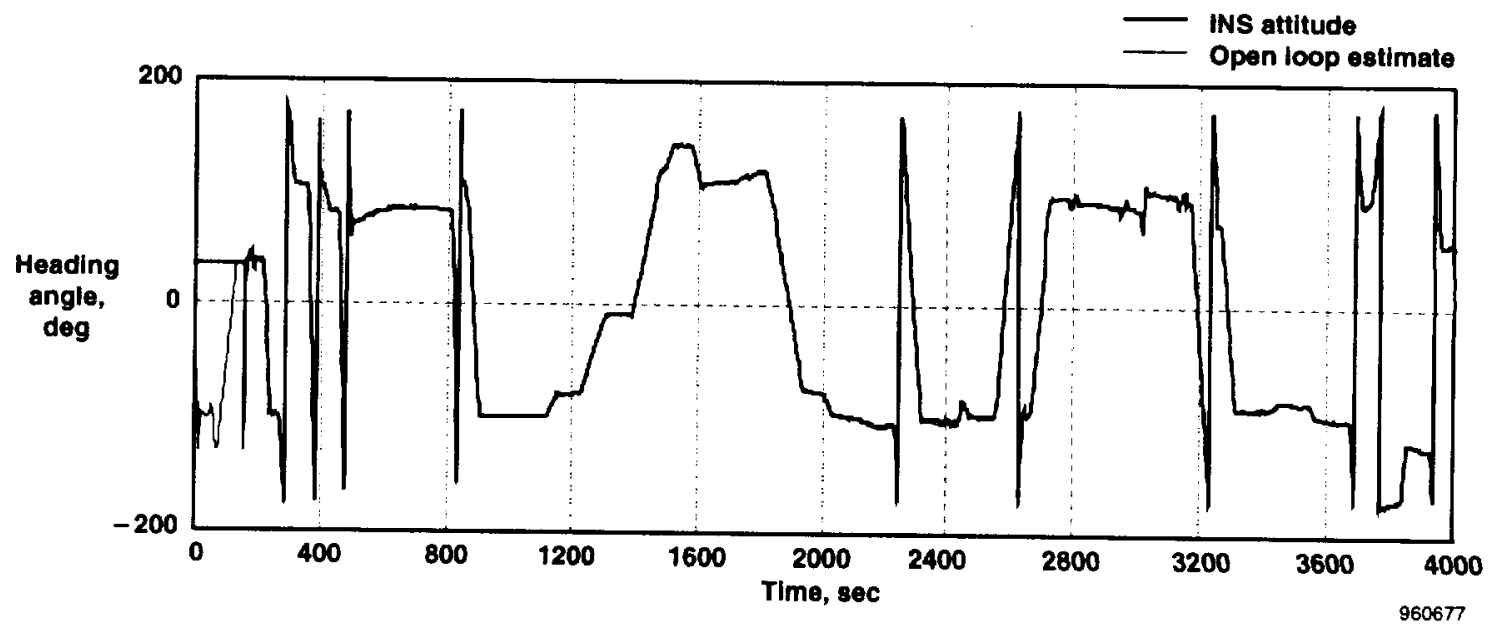

(c) Heading angle.

Figure 12. SRA flight 540 Euler angle comparisons, INS to closed-loop estimates. 


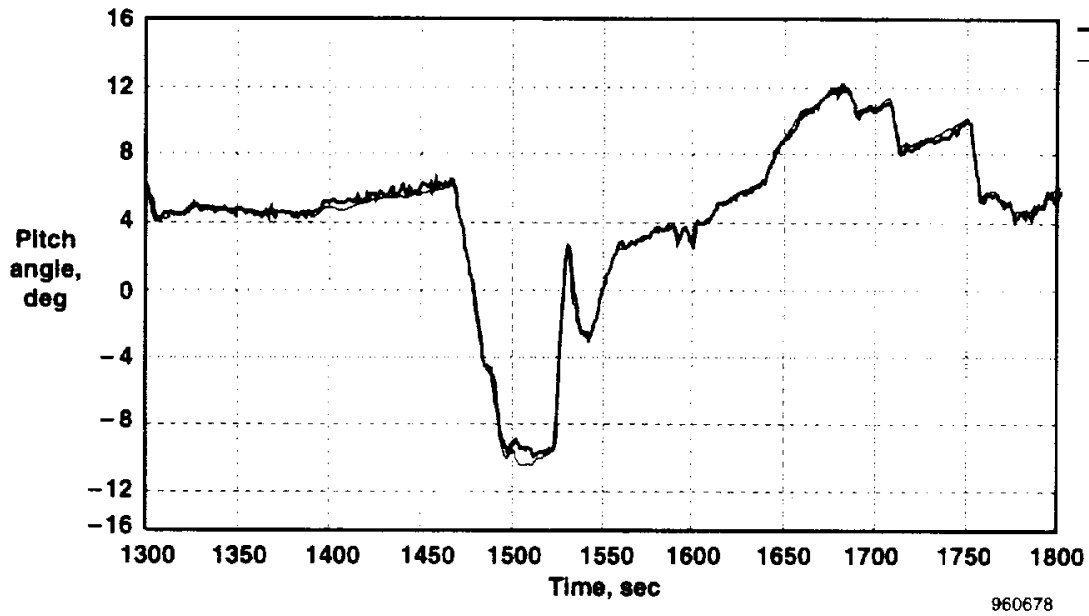

INS attitude

Closed loop estimate

(a) Pitch angle.

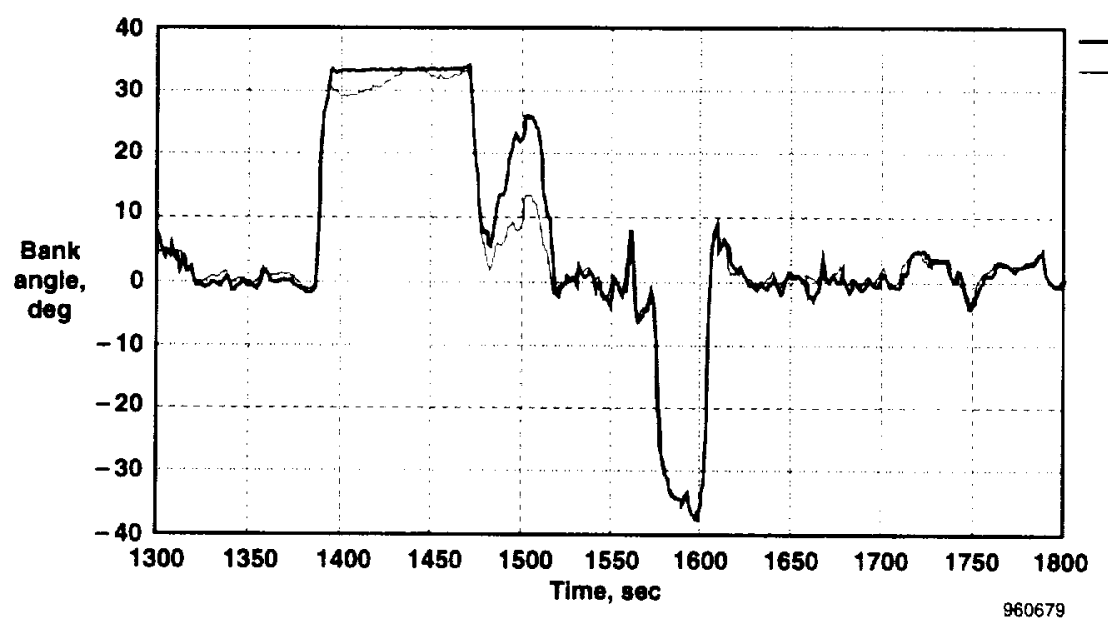

INS attltude

Closed loop estimate

(b) Bank angle.

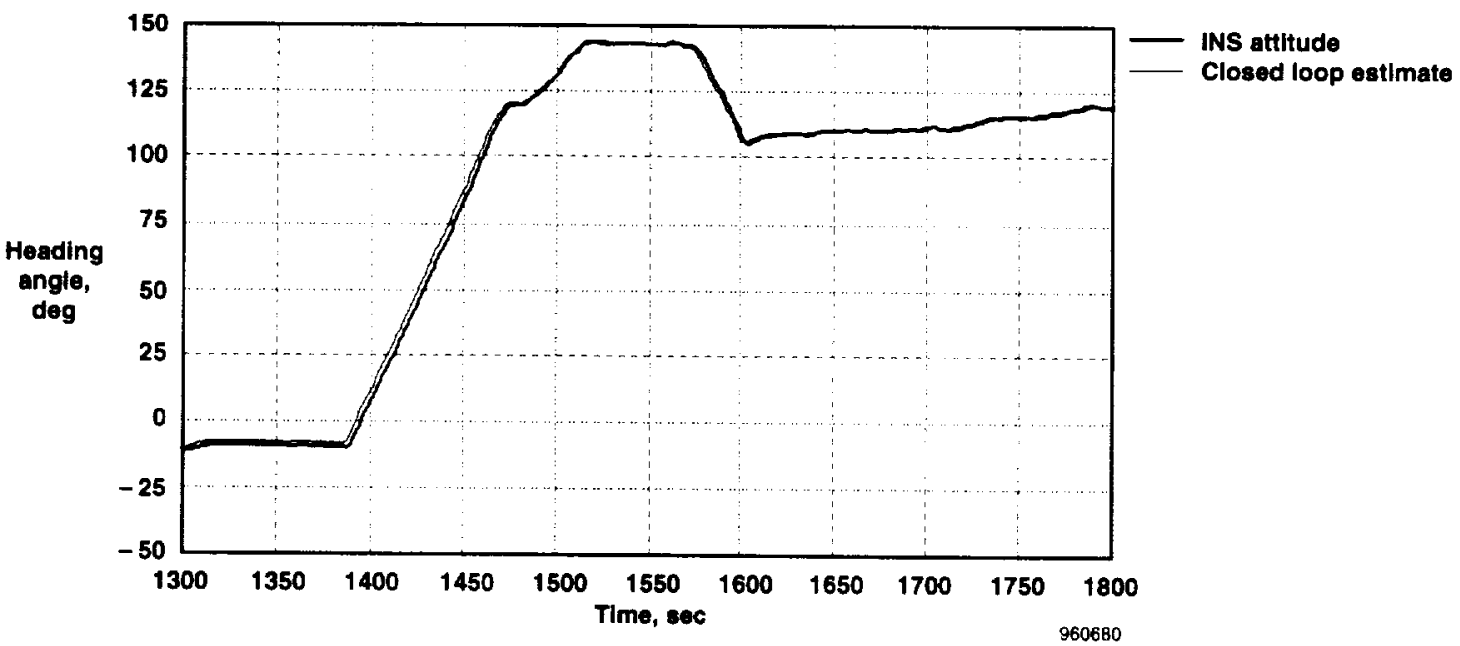

(c) Heading angle.

Figure 13. SRA flight 540, expanded scale Euler angle comparisons, INS to closed-loop estimates. 


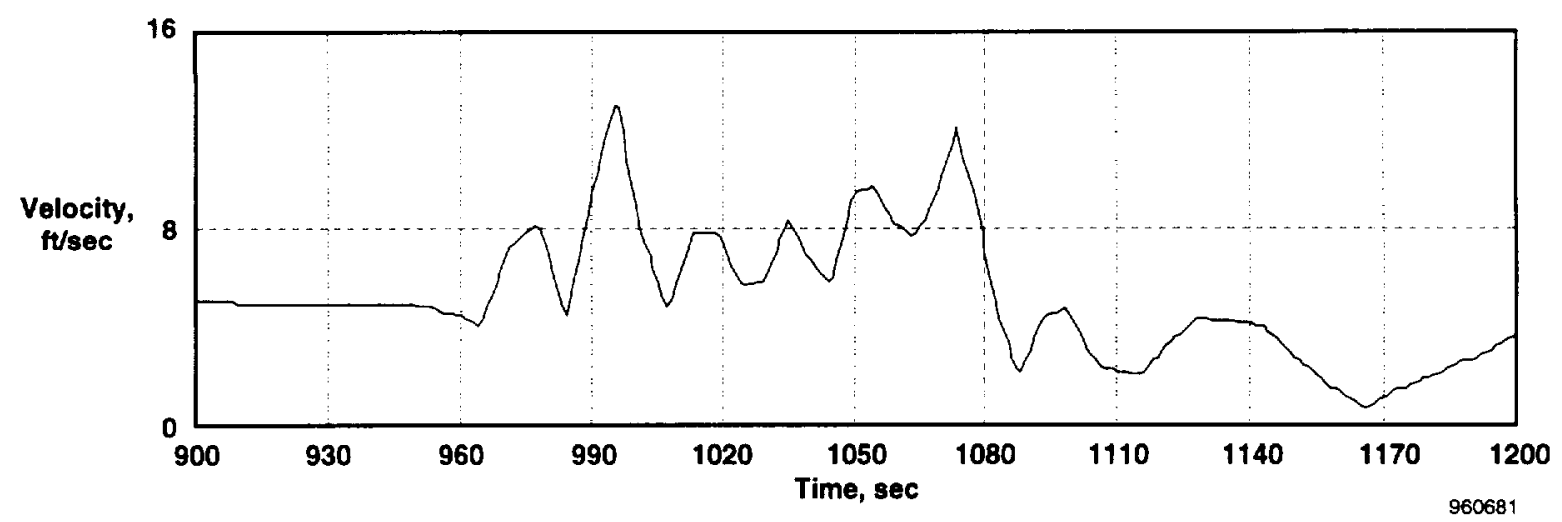

(a) Northerly wind component

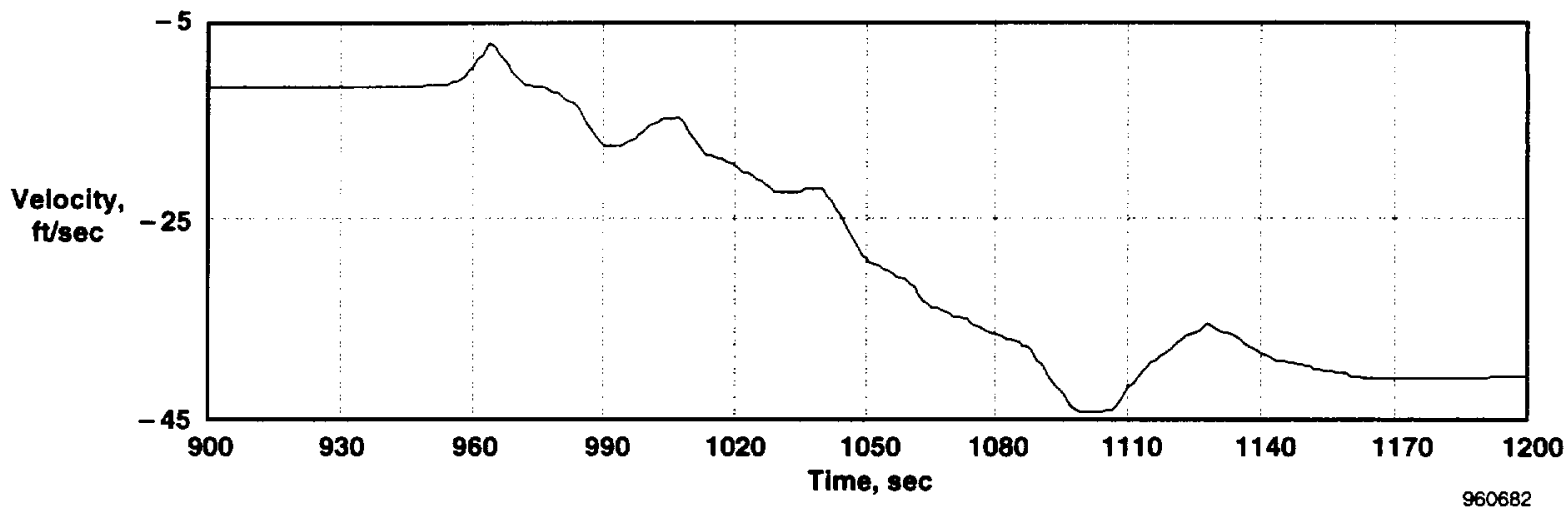

(b) Easterly wind component

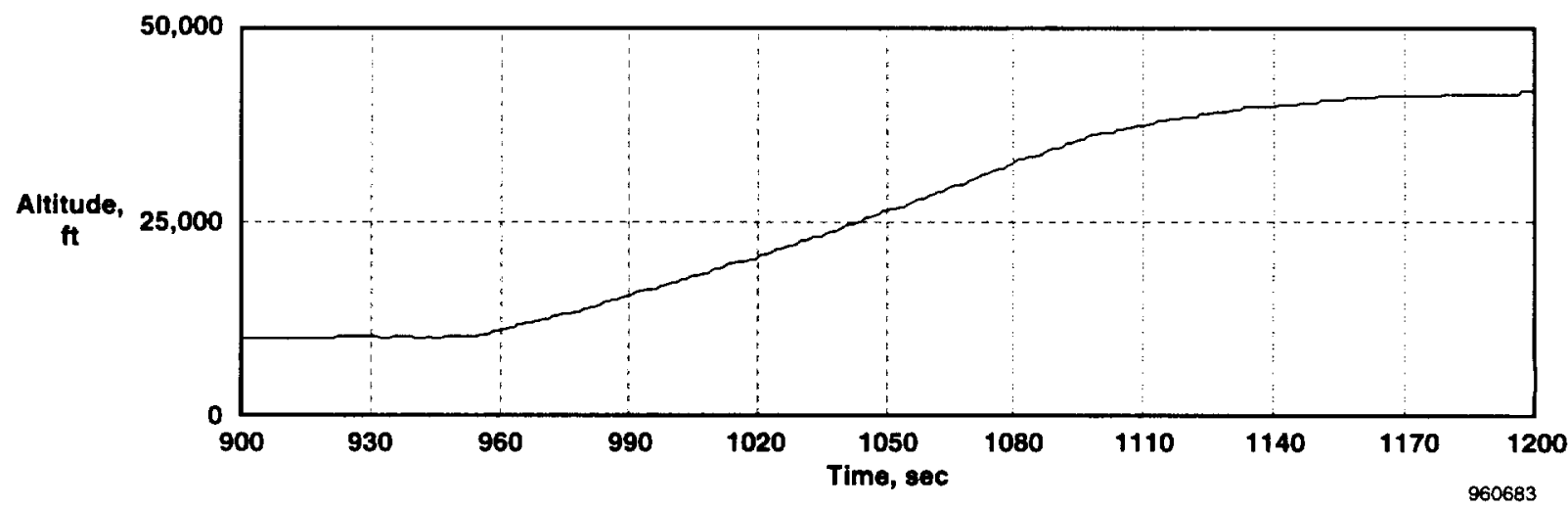

(c) Altitude

Figure 14. SRA flight 540 wind shear components and altitude time histories. 
Figure 15 shows the resulting estimates. Clearly, the stability of the computations has not been affected, but small changes in the attitude estimates are noted. The magnitudes of the induced errors in the pitch and heading angles are within the specified accuracy requirements of the program (less than $0.5^{\circ}$ for pitch and $2.0^{\circ}$ for heading). The accuracy limits are exceeded slightly for the bank angle, with induced errors as large as $2.5^{\circ}$ occurring. But as mentioned previously, because no roll information was provided by the single-axis compass, this result is not significant. Use of a three-axis magnetic-field measurement would likely reduce the bank angle errors to within the accuracy requirement limits. To be safe, if significant wind shears along the flightpath are anticipated, then wind tables from prelaunch balloon soundings could be loaded into the flight computer and used in the attitude computations.

\section{Summary and Concluding Remarks}

A low-cost strap-down architecture has been developed to estimate closed-loop attitudes for the ultrahigh altitude flight test experiment. In this system, body-axis observations of the airspeed and magneticfield vectors are compared with the measured inertial velocity and magnetic-field datum vectors to provide a "virtually inertial" reference that is used to infer attitude error. This error is then fed back to correct and stabilize the rate-gyro integration. The system has the stability of gimballed systems, but relies on strap-down components to gather the required information.

The system performance was analyzed for the ultrahigh altitude vehicle launch trajectory using Monte Carlo error simulations. These simulations verified that

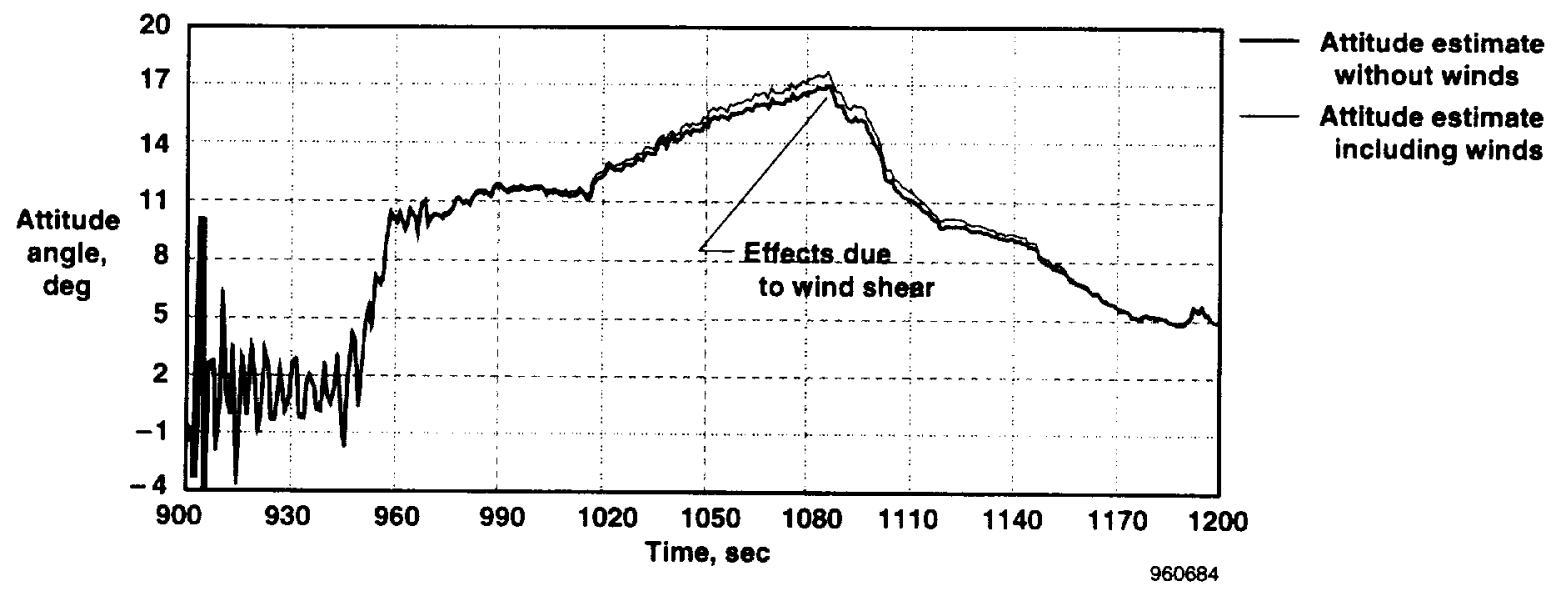

(a) Pitch angle.

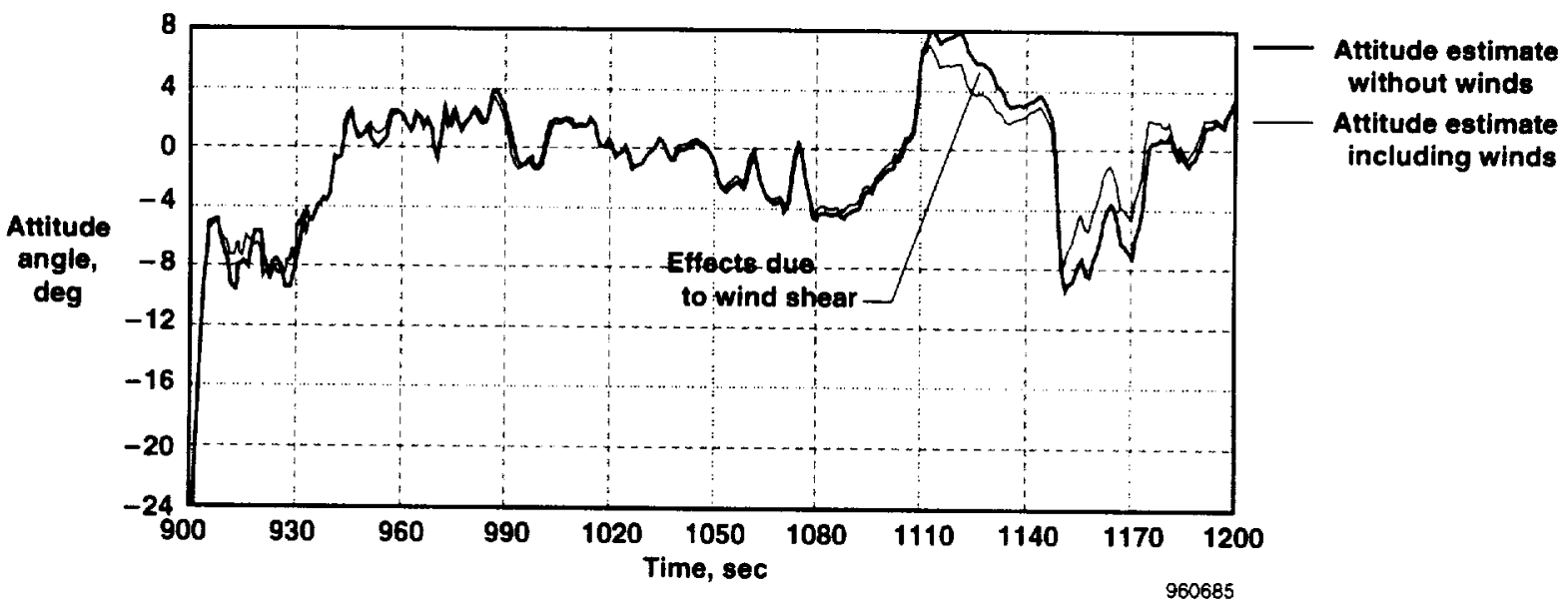

(b) Bank angle.

Figure 15. SRA flight 540, the effects of wind shear on the attitude estimates. 


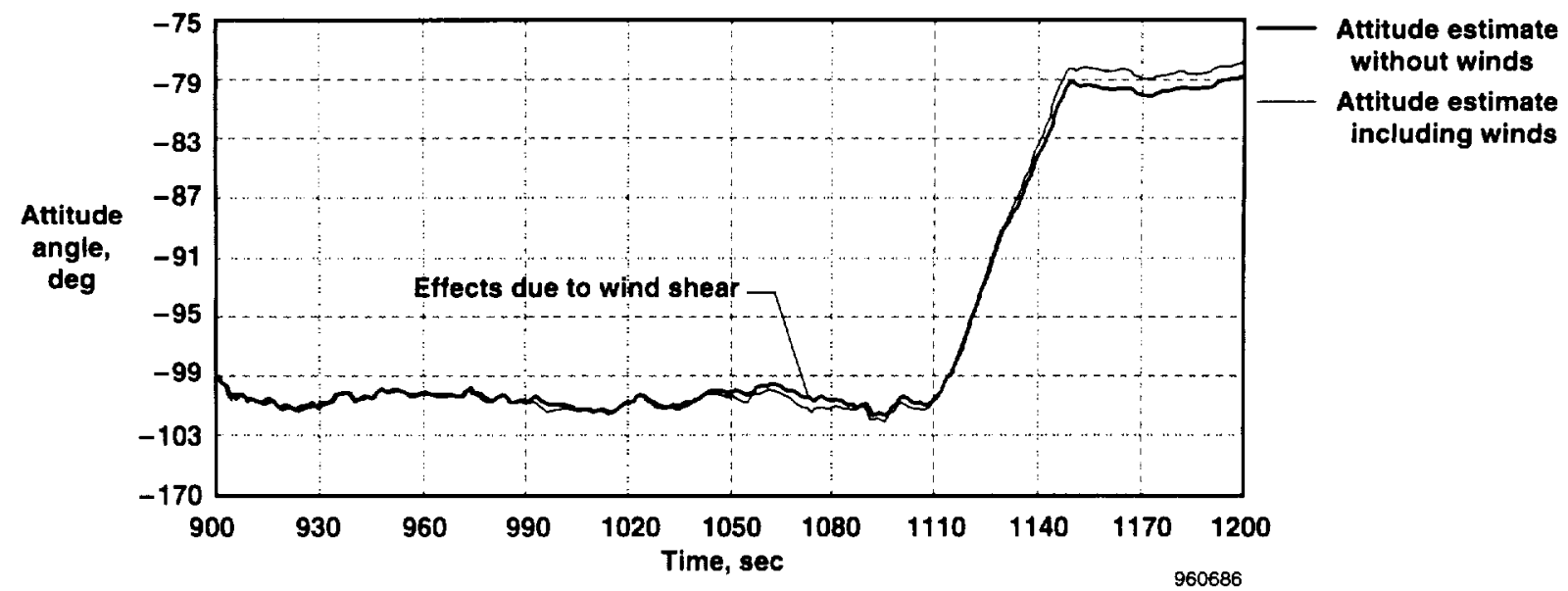

(c) Heading angle.

Figure 15. Concluded.

two vector measurements, magnetic field and velocity, are required to achieve complete three-attitude stability. Based on the accuracy requirements of the ultrahigh altitude vehicle, the simulations were used to establish accuracy requirements for the basic measurements. These requirements are not stringent and can easily be achieved using standard instrumentation. The two independent attitude references completely close the loop on the error equation so that a stable set of (linearized) error equations results and the errors of this system will not grow with time.

The effectiveness of the estimating algorithm under real flight conditions was demonstrated using data derived from the NASA Dryden Flight Research Center, Systems Research Aircraft flight tests. The algorithm was applied to data from several flights with flight conditions to a maximum $30^{\circ}$ pitch attitude with good results. Effects of wind shears on the attitude estimate were evaluated using these data, and it was concluded that the winds can be safely ignored by the estimating algorithm in most cases. To be safe, if significant wind shears along the flightpath are anticipated, then wind tables from prelaunch balloon soundings could be loaded into the flight computer and used in the attitude computations.

\section{References}

'Drela, Mark, "Transonic Low-Reynolds Number Airfoils," Journal of Aircraft, vol. 29, no. 6, Nov.-Dec. 1992.
${ }^{2}$ Toot, Peggy L., "Summary of Experimental Testing of a Transonic Low Reynolds Number Airfoil," Low Reynolds Number Aerodynamics Conference, Notre Dame, June 1989, pp. 369-380.

${ }^{3}$ Murray, James F., Moes, Timothy R., Norlin, Ken, Bauer, Jeff, Geenen, Robert J., and Moulton, Bryan J., "A Piloted Simulation Study of a Balloon-Assisted Deployment of an Aircraft at High Altitude," NASA TM-104245, Jan. 1992.

${ }^{4}$ Regan, Frank J. and Anandakrishnan, Staya M., "ReEntry Vehicle Dynamics," AIAA Education Series, American Institute of Aeronautics and Astronautics, New York, 1993.

5James, F., Monte Carlo Theory and Practice, Reports on Progress in Physics, vol. 43, Sept. 1980, pp. 1145-1189.

${ }^{6}$ Etkin, Bernard, Dynamics of Atmospheric Flight, John Wiley and Sons, New York, 1972.

${ }^{7}$ Langel, L. A., Mundt, W., Barraclough, D. R., Barton, C. E., Golovkov, V. P., Hood, P. J., Lowes, F. J., Peddie, N. W., Qi, GUI-Zhong, and Quinn, J. M., "International Geomagnetic Reference Field," 1991 revision, Journal of Geomagnetism and Geoelectricity, vol. 43, no. 12, 1991, pp. 1007-1012.

${ }^{8}$ Battin, Richard H., "An Introduction to the Mathematics and Methods of Astrodynamics," AIAA Education Series, American Institute of Aeronautics and Astronautics, New York, 1987.

'Rade, Lennart, and Westergren, Bertil, Beta Mathematics Handbook, 2nd ed., CRC Press, Boca Raton, FL, 1990. 
${ }^{10}$ Ehernberger, L. J., Haering, Edward A., Jr., Lockhart, Mary G., and Teets, Edward H., "Atmospheric Analysis for Airdata Calibration on Research Aircraft," AIAA Paper 92-0293, Jan. 1992.

${ }^{1}$ Meditch, J. S., Stochastic Optimal Linear Estimation and Control, McGraw-Hill Book Co., Inc., New York, 1969.

${ }^{12}$ Franklin, Gene F. and Powell, J. David, Digital Control of Dynamic Systems, Addison Wesley Publishing Co., Reading, Massachusetts, 1980.
${ }^{13}$ Whitmore, Stephen A., Davis, Roy J., and Fife, John Michael, In-Flight Demonstration of a Real-Time Flush Air Data Sensing (RT-FADS) System, NASA TM-104314, Oct. 1995.

${ }^{14}$ F/TF-18A Naval Air Training and Operating Procedures Standards (NATOPS) Manual, Reference \# A1-F18AA-NFM-O00, Feb. 1980.

${ }^{15}$ Haering, Edward A., Jr. and Whitmore, Stephen A., FORTRAN Program for the Analysis of Ground-Based Radar Data; Derivations and Usage, Version 6.2, NASA TM-3430, 1995. 

Public reporting burden for this coliection of intormation is estimated to average 1 hour per response, including the time for reviewing instructions, searching existing data sources, gathering and maintaining the data neded, and completing and reviewing the collection of information. Send comments regarding this burden estimate or any other aspect of this collection of intormation, including suggestions for reducing this burden, to Washington Headquarters Services. Directorate for Intormation Operations and Reports, 1215 Jefterson Davis Highway. Suite 1204 , Artington, VA 22202-4302, and to the Otfice of Management and Budget, Paperwork Feduction Project (0704-0188). Washington. DC 20503.

\begin{tabular}{|l|l|l}
\hline 1. AGENCY USE ONLY (Leave blank) & $\begin{array}{l}\text { 2. REPORT DATE } \\
\text { January } 1997\end{array}$ & $\begin{array}{l}\text { 3. REPORT TYPE AND DATES COVERED } \\
\text { Technical Memorandum }\end{array}$
\end{tabular}

4. TITLE AND SUBTITLE

Development of a Closed-Loop Strap Down Attitude System for an Ultrahigh Altitude Flight Experiment

6. AUTHOR(S)

Stephen A. Whitmore, Mike Fife, and Logan Brashear
8. PERFORMING ORGANIZATION

P.O. Box 273

Edwards, California 93523-0273 REPORT NUMBER

$\mathrm{H}-2140$

5. FUNDING NUMBERS

WU 537-10-40

H-2140

10. SPONSORINGMONITORING AGENCY REPORT NUMBER

NASA TM-4775

Washington, DC 20546-0001

\section{NASA TM-4775}

11. SUPPLEMENTARY NOTES

Presented at the 35th Aerospace Sciences and Exhibit, January 6-9, 1997, Reno, Nevada.

\begin{tabular}{l|l|}
\hline 12a. DISTRIBUTIONAVAILABILTTY STATEMENT & 12b. DISTRIBUTION CODE \\
Unclassified-Unlimited & \\
Subject Category 06 & \\
\hline 13. ABSTRACT (Maximum 200 words) &
\end{tabular}

A low-cost attitude system has been developed for an ultrahigh altitude flight experiment. The experiment uses a remotely piloted sailplane, with the wings modified for flight at altitudes greater than $100,000 \mathrm{ft}$. Mission requirements deem it necessary to measure the aircraft pitch and bank angles with accuracy better than $1.0^{\circ}$ and heading with accuracy better than $5.0^{\circ}$. Vehicle cost restrictions and gross weight limits make installing a commercial inertial navigation system unfeasible. Instead, a low-cost attitude system was developed using strap down components. Monte Carlo analyses verified that two vector measurements, magnetic field and velocity, are required to completely stabilize the error equations. In the estimating algorithm, body-axis observations of the airspeed vector and the magnetic field are compared against the inertial velocity vector and a magnetic-field reference model. Residuals are fed back to stabilize integration of rate gyros. The effectiveness of the estimating algorithm was demonstrated using data from the NASA Dryden Flight Research Center Systems Research Aircraft (SRA) flight tests. The algorithm was applied with good results to a maximum $10^{\circ}$ pitch and bank angles. Effects of wind shears were evaluated and, for most cases, can be safely ignored.

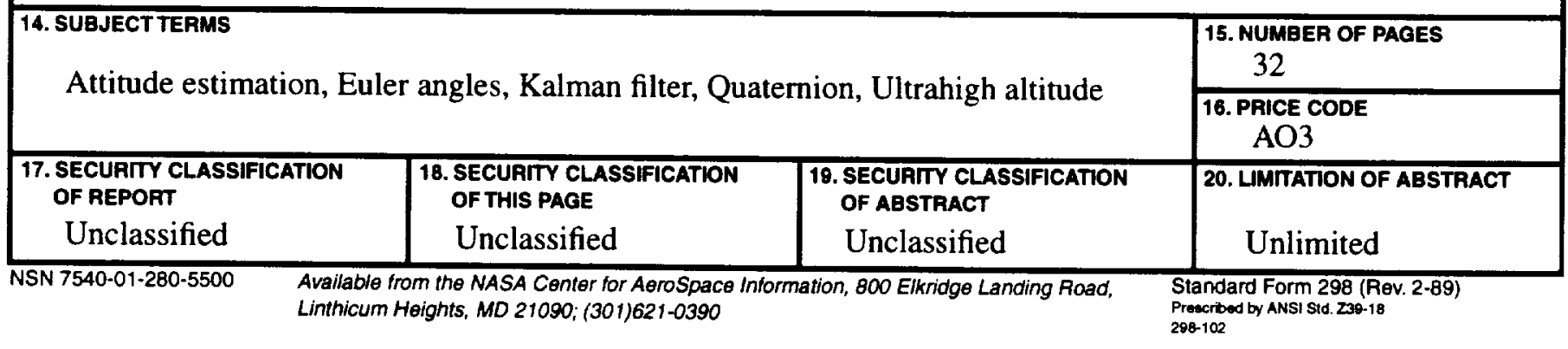

